TRANSACTIONS OF THE

AMERICAN MATHEMATICAL SOCIETY

Volume 364, Number 12, December 2012, Pages 6395-6424

S 0002-9947(2012)05549-8

Article electronically published on July 11, 2012

\title{
REPRESENTATIONS OF DYNAMICAL SYSTEMS ON BANACH SPACES NOT CONTAINING $l_{1}$
}

\author{
E. GLASNER AND M. MEGRELISHVILI
}

\begin{abstract}
For a topological group $G$, we show that a compact metric $G$-space is tame if and only if it can be linearly represented on a separable Banach space which does not contain an isomorphic copy of $l_{1}$ (we call such Banach spaces, Rosenthal spaces). With this goal in mind we study tame dynamical systems and their representations on Banach spaces.
\end{abstract}

\section{Contents}

1. Introduction

2. Topological background: Fragmentability and Baire 1 functions

3. Dynamical background: $G$-spaces and Banach representations

4. Banach space background and the dynamical BFT dichotomy

5. Tame dynamical systems

6410

6. The main results

6413

References

\section{INTRODUCTION}

1.1. Some important dichotomies. Rosenthal's celebrated dichotomy theorem asserts that every bounded sequence in a Banach space either has a weak Cauchy subsequence or admits a subsequence equivalent to the unit vector basis of $l_{1}$ (an $l_{1}$ sequence). Thus, a Banach space $V$ does not contain an $l_{1}$-sequence (equivalently, does not contain an isomorphic copy of $l_{1}$ ) if and only if every bounded sequence in $V$ has a weak-Cauchy subsequence [38. In the present work we call a Banach space satisfying these equivalent conditions a Rosenthal space.

The theory of Rosenthal spaces is one of the cases where the interplay between Analysis and Topology gives rise to many deep results. Our aim is to show the relevance of Topological Dynamics in this interplay. In particular, we examine when a dynamical system can be represented on a Rosenthal space, and we show that being tame is a complete characterization of such systems.

Received by the editors April 16, 2008 and, in revised form, November 9, 2009 and January $21,2011$.

2010 Mathematics Subject Classification. Primary 37Bxx, 54H20, 54H15, 46-xx.

Key words and phrases. Baire one function, Banach representation of dynamical systems, enveloping semigroup, fragmentability, Rosenthal's dichotomy, Rosenthal's compact, Tame system.

The first author's research was partially supported by BSF (Binational USA-Israel) grant no. 2006119.

(C)2012 American Mathematical Society Reverts to public domain 28 years from publication 
First we recall some results and ideas. The following dichotomy is a version of a result of Bourgain, Fremlin and Talagrand [2] (as presented in the book of Todorčević [43, Proposition 1 of Section 13).

Fact 1.1 (BFT dichotomy). Let $X$ be a Polish space and let $\left\{f_{n}\right\}_{n=1}^{\infty} \subset C(X)$ be a sequence of real-valued functions which is pointwise bounded (i.e. for each $x \in X$ the sequence $\left\{f_{n}(x)\right\}_{n=1}^{\infty}$ is bounded in $\left.\mathbb{R}\right)$. Let $K$ be the pointwise closure of $\left\{f_{n}\right\}_{n=1}^{\infty}$ in $\mathbb{R}^{X}$. Then either $K \subset \mathcal{B}_{1}(X)$, where $\mathcal{B}_{1}(X)$ denotes the space of all real-valued Baire 1 functions on $X$, or $K$ contains a homeomorphic copy of $\beta \mathbb{N}$.

In [13, Theorem 3.2] the following dynamical dichotomy, in the spirit of the Bourgain-Fremlin-Talagrand theorem, was established.

Fact 1.2 (A dynamical BFT dichotomy). Let $X$ be a compact metric dynamical $G$ system and let $E(X)$ be its enveloping semigroup. We have the following dichotomy. Either

(1) $E(X)$ is a separable Rosenthal compactum, hence with cardinality card $E(X) \leq 2^{\aleph_{0}}$, or

(2) $E(X)$ contains a homeomorphic copy of $\beta \mathbb{N}$; hence card $E(X)=2^{2^{\aleph_{0}}}$.

In [10] a compact metric dynamical system is called tame if the first alternative occurs, i.e. if $E(X)$ is a Rosenthal compactum. By [2, every Rosenthal compactum is a Fréchet space (and in particular its topology is determined by the converging sequences). Thus, either $E(X)$ (although not necessarily metrizable) has a nice topological structure, or it is as unruly as possible containing a copy of $\beta \mathbb{N}$. As to the metrizability of $E(X)$, recent results in [13] and [16] assert that $E(X)$ is metrizable iff the metric compact $G$-space $X$ is hereditarily nonsensitive (HNS) iff $X$ is Asplund representable (see Section 3.3.2). A Banach space $V$ is an Asplund space if the dual of every separable Banach subspace is separable (see Remarks 2.2(4)). Reflexive spaces and spaces of the type $c_{0}(\Gamma)$ are Asplund.

1.2. The main results and related facts. The main result of the present work is the following:

Theorem 1.3. Let $X$ be a compact metric $G$-space. The following conditions are equivalent:

(1) $(G, X)$ is a tame $G$-system.

(2) $(G, X)$ is representable on a separable Rosenthal Banach space.

This theorem continues a series of recent results which link dynamical properties of $G$-systems (such as WAP and HNS) to their representability on "good" Banach spaces (Reflexive and Asplund respectively). See Sections 1.3 and 3.3 below for more details.

One of the important questions in Banach space theory until the mid 1970s was how to construct a separable Rosenthal space which is not Asplund. The first examples were constructed independently by James [21] and Lindenstrauss and Stegall [27. In view of Theorem 1.3 we now see that a fruitful way of producing such distinguishing examples comes from dynamical systems. Just consider a compact metric tame $G$-system which is not HNS (see e.g. Remarks 5.10 below) and then apply Theorem 1.3 .

In order to get a better perspective on the position of tame systems in the hierarchy of dynamical systems we remind the reader of some enveloping semigroup 
characterizations. For a recent review of enveloping semigroup theory we refer to 12. A compact $G$-space $X$ is WAP (weakly almost periodic) if and only if its enveloping semigroup $E(X) \subset X^{X}$ consists of continuous maps (Ellis and Nerurkar [7]). Recently the following characterization of tameness was established.

Fact 1.4 (16]). A compact metric dynamical $G$-system $X$ is tame if and only if every element of $E(X)$ is a Baire class 1 function (equivalently, has the point of continuity property) from $X$ to itself.

A function $f: X \rightarrow Y$ has the point of continuity property if for every closed nonempty subset $A$ of $X$ the restriction $\left.f\right|_{A}: A \rightarrow Y$ has a point of continuity. For compact $X$ and metrizable $Y$ it is equivalent to the fragmentability (see Section 2 and Lemma 2.5) of the function $f$. The topological concept of fragmentability comes in fact from Banach space theory (Jayne-Rogers [23]). For dynamical applications of fragmentability, we refer to [28, 29, 30, 13, 14, 15.

Fact 1.4 suggests the following general definition.

Definition 1.5. Let $X$ be a (not necessarily metrizable) compact $G$-space. We say that $X$ is tame if for every element $p \in E(X)$ the function $p: X \rightarrow X$ is fragmented.

The class of tame dynamical systems contains the class of HNS systems and hence also WAP systems. Indeed, as we already mentioned, every function $p: X \rightarrow X$ $(p \in E(X))$ is continuous for WAP systems. As to the HNS systems they can be characterized as those $G$-systems where the family of maps $\{p: X \rightarrow X\}_{p \in E(X)}$ is a fragmented family (see Definition 2.7 and Fact 3.4 below). In particular, every individual $p: X \rightarrow X$ is a fragmented map. Thus, these enveloping semigroup characterizations yield a natural hierarchy of the three classes, WAP, HNS and Tame, of dynamical systems.

In [26, Köhler introduced the definition of regularity for cascades (i.e. $\mathbb{Z}$-dynamical systems) in terms of independent sequences and, using results of BourgainFremlin-Talagrand, has shown that her definition can be reformulated in terms of $l_{1}$-sequences. Extending Köhler's definition to arbitrary topological groups $G$, we say that a compact $G$-space $X$ is regular if, for any $f \in C(X)$, the orbit $f G$ does not contain an $l_{1}$-sequence (in other words, the second alternative is ruled out in the Rosenthal dichotomy). As we will see later, in Corollary [5.8, a $G$-system is regular if and only if it is tame (for metrizable $X$ this fact was established in [10]).

In Theorem 6.10 we give a characterization of Rosenthal representable $G$-systems. As a particular case (for trivial $G$ ) we get a topological characterization of compact spaces which are homeomorphic to weak* compact subsets in the dual of Rosenthal spaces. A well-known result characterizes Rosenthal spaces as those Banach spaces whose dual has the weak Radon-Nikodým property [42, Corollary 7.3.8]. It is therefore natural to call such a compact space a weakly Radon-Nikodým compactum (WRN). Theorem 6.5 gives a simple characterization in terms of fragmentability. Namely, a compact space $X$ is WRN iff there exists a bounded subset $F \subset C(X)$ such that the pointwise closure of $F$ in $\mathbb{R}^{X}$ consists of fragmented maps from $X$ to $\mathbb{R}$ and $F$ separates points of $X$.

Theorem [6.10 is related to yet another characterization of Rosenthal Banach spaces. Precisely, let $V$ be a Banach space with dual $V^{*}$ and second dual $V^{* *}$. One may consider the elements of $V^{* *}$ as functions on the weak* compact unit ball $B^{*}:=B_{V^{*}} \subset V^{*}$. While the elements of $V$ are clearly continuous on $B^{*}$ it is not 
true in general for elements from $V^{* *}$. By a result of Odell and Rosenthal [35], a separable Banach space $V$ is Rosenthal iff every element $v^{* *}$ from $V^{* *}$ is a Baire one function on $B^{*}$. More generally, E. Saab and P. Saab [41] show that $V$ is Rosenthal iff every element of $V^{* *}$ has the point of continuity property when restricted to $B^{*}$. Equivalently, every restriction of $v^{* *}$ to a bounded subset $M$ is fragmented as a function $\left(M, w^{*}\right) \rightarrow \mathbb{R}$ (see Fact 4.11 below).

Answering a question of Talagrand [42, Problem 14-2-41], R. Pol [36] gave an example of a separable compact Rosenthal space $K$ which cannot be embedded in $\mathcal{B}_{1}(X)$ for any compact metrizable $X$. We say that a compact space $K$ is strongly Rosenthal if it is homeomorphic to a subspace of $\mathcal{B}_{1}(X)$ for a compact metrizable $X$. We say that a compact space $K$ is admissible if there exists a metrizable compact space $X$ and a bounded subset $Z \subset C(X)$ such that the pointwise closure $\operatorname{cls}_{p}(Z)$ of $Z$ in $\mathbb{R}^{X}$ consists of Baire 1 functions and $K \subset \operatorname{cls}_{p}(Z)$. Clearly every admissible compactum is strongly Rosenthal. We do not know whether these two classes of compact spaces coincide. Note that the enveloping semigroup $K:=E(X)$ of a compact metrizable $G$-space $X$ is admissible iff $(G, X)$ is tame (Proposition 6.15).

As another consequence of our analysis we show that a compact space $K$ is an admissible Rosenthal compactum iff it is homeomorphic to a weak* closed bounded subset in the second dual of a separable Rosenthal Banach space $V$ (Theorem 6.16).

Remark 1.6. We note that the main results of our work remain true for semigroup actions once some easy modifications are introduced.

Remark 1.7. The attentive reader will not fail to detect the major importance to our work of the papers [4, 2], and the book [42].

1.3. The hierarchy of Banach representations. In Table 1 we encapsulate some features of the trinity: dynamical systems, enveloping semigroups, and Banach representations. Let $X$ be a compact metrizable $G$-space and $E(X)$ denote the corresponding enveloping semigroup. The symbol $f$ stands for an arbitrary function in $C(X)$ and $f G=\{f \circ g: g \in G\}$ denotes its orbit. Finally, cls $(f G)$ is the pointwise closure of $f G$ in $\mathbb{R}^{X}$.

TABLE 1. The hierarchy of Banach representations

\begin{tabular}{|l|l|l|l|}
\hline & Dynamical characterization & Enveloping semigroup & Banach representation \\
\hline WAP & $\operatorname{cls}(f G)$ is a subset of $C(X)$ & Every element is continuous & Reflexive \\
\hline HNS & $\operatorname{cls}(f G)$ is metrizable & $E(X)$ is metrizable & Asplund \\
\hline Tame & $\operatorname{cls}(f G)$ is Fréchet & Every element is Baire 1 & Rosenthal \\
\hline
\end{tabular}

\section{Topological background: Fragmentability AND BAIRE 1 FUNCTIONS}

Let $X$ be a topological space and $A \subset X$. We say that $A$ is relatively compact in $X$ if the closure cls $(A)$ is a compact subset of $X$. We say that $A$ is sequentially precompact in $X$ if every sequence in $A$ has a subsequence which converges in $X$. Compact space will mean compact and Hausdorff.

The following definition is a generalized version of fragmentability.

Definition $2.1([22,28])$. Let $(X, \tau)$ be a topological space and $(Y, \mu)$ a uniform space. We say that $X$ is $(\tau, \mu)$-fragmented by a (typically not continuous) function 
$f: X \rightarrow Y$ if for every nonempty subset $A$ of $X$ and every entourage $\varepsilon \in \mu$ there exists an open subset $O$ of $X$ such that $O \cap A$ is nonempty and the set $f(O \cap A)$ is $\varepsilon$-small in $Y$. We also say in that case that the function $f$ is fragmented. Notation: $f \in \mathcal{F}(X, Y)$, whenever the uniformity $\mu$ is understood. If $Y=\mathbb{R}$, then we write simply $\mathcal{F}(X)$.

Remarks 2.2. (1) In Definition 2.1 when $Y=X, f=i d_{X}$ and $\mu$ is a metric uniform structure, we get the usual definition of fragmentability in the sense of Jayne and Rogers 23. Implicitly it already appears in a paper of Namioka and Phelps [34.

(2) It is enough to check the condition of Definition 2.1 only for closed subsets $A \subset X$ and for $\varepsilon \in \mu$ from a subbase $\gamma$ of $\mu$ (that is, the finite intersections of the elements of $\gamma$ form a base of the uniform structure $\mu$ ).

(3) Namioka's joint continuity theorem 32 implies that every weakly compact subset $K$ of a Banach space is (weak, norm)-fragmented (that is, $i d_{K}$ : $(K$, weak $) \rightarrow(K$, norm $)$ is fragmented $)$.

(4) Recall that a Banach space $V$ is an Asplund space if the dual of every separable Banach subspace is separable, iff every bounded subset $A$ of the dual $V^{*}$ is (weak*, norm)-fragmented, iff $V^{*}$ has the Radon-Nikodým property. Reflexive spaces and spaces of the type $c_{0}(\Gamma)$ are Asplund. For more details, cf. [3, 9, 33.

(5) A Banach space $V$ is Rosenthal if and only if every bounded subset $A$ of the dual $V^{*}$ is (weak* topology, weak uniformity)-fragmented. This follows by Proposition 4.12,

Recall that $f: X \rightarrow Y$ is barely continuous, 31, if for every nonempty closed subset $A \subset X$, the restricted map $\left.f\right|_{A}$ has at least one point of continuity. Following [42, Section 14] the set of barely continuous functions $f: X \rightarrow \mathbb{R}$ is denoted by $B_{r}^{\prime}(X)$.

Lemma 2.3. (1) Every barely continuous $f$ is fragmented.

(2) Let $\alpha: X \rightarrow Y$ be a continuous map. If $f: Y \rightarrow(Z, \mu)$ is a fragmented map, then the composition $f \circ \alpha: X \rightarrow(Z, \mu)$ is also fragmented.

(3) Let $p: X \rightarrow Y$ be a map from a topological space $X$ into a compact space $Y$. Suppose that $\left\{f_{i}: Y \rightarrow Z_{i}\right\}_{i \in I}$ is a system of continuous maps from $Y$ into Hausdorff uniform spaces $Z_{i}$ such that it separates points of $Y$ and $f_{i} \circ p \in \mathcal{F}\left(X, Z_{i}\right)$ for every $i \in I$. Then $p \in \mathcal{F}(X, Y)$.

(4) Let $(X, \tau)$ and $\left(X^{\prime}, \tau^{\prime}\right)$ be compact spaces, and let $(Y, \mu)$ and $\left(Y^{\prime}, \mu^{\prime}\right)$ be uniform spaces. Suppose that $\alpha: X \rightarrow X^{\prime}$ is a continuous onto map, $\nu:(Y, \mu) \rightarrow\left(Y^{\prime}, \mu^{\prime}\right)$ is uniformly continuous, $\phi: X \rightarrow Y$ and $\phi^{\prime}: X^{\prime} \rightarrow Y^{\prime}$ are maps such that the diagram

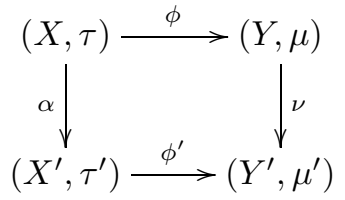

commutes. If $X$ is fragmented by $\phi$, then $X^{\prime}$ is fragmented by $\phi^{\prime}$.

(5) Let $\alpha: X \rightarrow X^{\prime}$ be a continuous onto map between compact spaces. Assume that $(Y, \mu)$ is a uniform space, $f: X \rightarrow Y$ and $f^{\prime}: X^{\prime} \rightarrow Y$ are maps such that $f^{\prime} \circ \alpha=f$. Then $f$ is a fragmented map iff $f^{\prime}$ is a fragmented map. 
(6) If $f: X \rightarrow Y$ is fragmented, where $(X, \tau)$ is a Baire space and $(Y, \rho)$ is a pseudometric space, then $f$ is continuous at the points of a dense $G_{\delta}$ subset of $X$.

Proof. (1): is straightforward.

(2): Let $A$ be a nonempty subset of $X$ and let $\varepsilon \in \mu$. Choose an open subset $O$ in $Y$ such that $\alpha(A) \cap O$ is nonempty and $f(\alpha(A) \cap O)$ is $\varepsilon$-small. Since $\alpha\left(A \cap \alpha^{-1}(O)\right)=$ $\alpha(A) \cap O$ we get that $A \cap \alpha^{-1}(O)$ is nonempty and $(f \circ \alpha)\left(A \cap \alpha^{-1}(O)\right)$ is $\varepsilon$-small in $Y$. This completes the proof because $\alpha^{-1}(O)$ is open in $X$ by the continuity of $\alpha$.

(3): Consider the weak uniformity $\mu_{w}$ on $Y$ generated by the system $\left\{f_{i}: Y \rightarrow\right.$ $\left.Z_{i}\right\}_{i \in I}$. Since this system separates the points of $Y$ and each $Z_{i}$ is a Hausdorff uniform space we get that $\mu_{w}$ is a Hausdorff uniformity on $Y$. Furthermore $\mu_{w}$ is continuous on $Y$. Now it is clear that $\mu_{w}$ coincides with the unique compatible uniformity on the compact space $Y$. The system of entourages

$$
\left\{\left(f_{i}^{-1} \times f_{i}^{-1}\right)\left(\varepsilon_{i}\right) \mid \varepsilon_{i} \in \mu_{i}\right\}
$$

is a subbase of the uniformity $\mu_{w}$. Now use Remark $2.2(2)$.

(4): This is [13, Lemma 6.4], which in turn was inspired by Lemma 2.1 of Namioka's paper 33 .

(5): If $f^{\prime}$ is fragmented, then $f$ is fragmented by (2). If $f$ is fragmented, then $f^{\prime}$ is fragmented by (4) (with $Y=Y^{\prime}$ and $\nu=i d_{Y}$ ).

(6): For a fixed $\varepsilon>0$ consider

$$
O_{\varepsilon}:=\left\{\text { union of all } \tau \text {-open subsets } O \text { of } X \text { with } \operatorname{diam}_{\rho} f(O) \leq \varepsilon\right\} .
$$

The fragmentability implies that $O_{\varepsilon}$ is dense in $X$. Clearly $\bigcap\left\{O_{\frac{1}{n}}: n \in \mathbb{N}\right\}$ serves as the required dense $G_{\delta}$ subset of $X$.

2.1. Baire class one functions. Given two topological spaces $X$ and $Y$, a function $f: X \rightarrow Y$ is of Baire class 1 or more briefly Baire 1 if the inverse image of every open set in $Y$ is $F_{\sigma}$ (the union of countably many closed sets) in $X$. In general a Baire 1 function need not be the same as a limit of a sequence of continuous functions. The following results are well known. Mainly they are classical and come from R. Baire. See for example [5, 24, 16].

Lemma 2.4. (1) If $Y$ is metrizable and $\left\{f_{n}: X \rightarrow Y\right\}_{n \in \mathbb{N}}$ is a sequence of continuous functions converging pointwise to $f: X \rightarrow Y$, then $f$ is Baire 1.

(2) If $X$ is separable and metrizable, then a real-valued function $f: X \rightarrow \mathbb{R}$ is Baire 1 if and only if $f$ is a pointwise limit of a sequence of continuous functions.

We denote by $\mathcal{B}_{1}(X)$ the space of all real-valued Baire 1 functions on $X$ equipped with the pointwise convergence topology. That is, $\mathcal{B}_{1}(X)$ is a topological subspace of the product space $\mathbb{R}^{X}$.

As usual, a space is Baire if the intersection of any countable family of dense open sets is dense. Hereditarily Baire means that every closed subspace is a Baire space. 
Lemma 2.5. Let $(X, \tau)$ be a hereditarily Baire (e.g., Polish, or compact) space, $(Y, \rho)$ a pseudometric space. Consider the following assertions:

(a) $X$ is $(\tau, \rho)$-fragmented by $f: X \rightarrow Y$;

(b) $f$ is barely continuous;

(c) $f$ is of Baire class 1.

(1) Then $(a) \Leftrightarrow(b)$.

(2) If $X$ is Polish and $Y$ is a separable metric space, then $(a) \Leftrightarrow(b) \Leftrightarrow(c)$.

Proof. For (a) $\Leftrightarrow$ (b) use Lemma 2.3 .

The equivalence (b) $\Leftrightarrow(\mathrm{c})$ for Polish $X$ and separable metric space $Y$ is well known (see [24, Theorem 24.15]) and goes back to Baire.

Corollary 2.6. (1) $\mathcal{F}(X)=B_{r}^{\prime}(X)$ for every compact space $X$.

(2) $\mathcal{F}(X)=\mathcal{B}_{1}(X)=B_{r}^{\prime}(X)$ for every Polish space $X$.

2.2. Fragmented families. The following definition was introduced in [13] and independently in the Ph.D. Thesis of M.M. Guillermo [19].

Definition 2.7. (1) We say that a family of functions $F=\{f:(X, \tau) \rightarrow$ $(Y, \mu)\}$ is fragmented if the condition of Definition 2.1(1) holds simultaneously for all $f \in F$. That is, $O \cap A$ is nonempty and $f(O \cap A)$ is $\varepsilon$-small for every $f \in F$. It is equivalent to saying that the mapping

$$
\pi_{\sharp}: X \rightarrow Y^{F}, \quad \pi_{\sharp}(x)(f)=f(x)
$$

is $\left(\tau, \mu_{u}\right)$-fragmented, where $\mu_{u}$ is the uniform structure of uniform convergence on the set $Y^{F}$ of all mappings from $F$ into $(Y, \mu)$.

(2) Analogously one can define the notion of a barely continuous family. The latter means that every closed nonempty subset $A \subset X$ contains a point $a \in A$ such that $F_{A}=\left\{\left.f\right|_{A}: f \in F\right\}$ is equicontinuous at $a$. If $\mu$ is pseudometrizable, then so is $\mu_{u}$. Therefore, if in addition $(X, \tau)$ is hereditarily Baire, then it follows by Lemma 2.5 (1) that $F$ is a fragmented family if and only if $F$ is a barely continuous family.

Fragmented families, like equicontinuous families, are stable under pointwise closures as the first assertion of the following lemma shows.

Lemma 2.8. (1) Let $F=\left\{f_{i}:(X, \tau) \rightarrow(Y, \mu)\right\}_{i \in I}$ be a fragmented family of functions. Then the pointwise closure cls ${ }_{p} F$ of $F$ in $Y^{X}$ is also a $(\tau, \mu)$ fragmented family.

(2) Let $\alpha: X \rightarrow X^{\prime}$ be a continuous onto map between compact spaces. Assume that $(Y, \mu)$ is a uniform space, $F:=\left\{f_{i}: X \rightarrow Y\right\}_{i \in I}$ and $F^{\prime}:=\left\{f_{i}^{\prime}: X^{\prime} \rightarrow\right.$ $Y\}$ are families such that $f_{i}^{\prime} \circ \alpha=f_{i}$ for every $i \in I$. Then $F$ is a fragmented family iff $F^{\prime}$ is a fragmented family.

Proof. (1) A straightforward " $3 \varepsilon$-argument".

(2) If $F^{\prime}$ is fragmented, then an obvious modification (for families) of Lemma 2.3. (2) shows that $F$ is fragmented.

If $F$ is fragmented, then this means that the identity map $(X, \tau) \rightarrow(X, \xi)$ is fragmented, where $\xi$ is the weak uniformity induced on the set $X$ by the natural map $X \rightarrow\left(Y^{F}, \mu_{u}\right)$ (see Definition 2.7). Analogously, the map $X^{\prime} \rightarrow\left(Y^{F^{\prime}}, \mu_{u}\right)$ induces the uniformity $\xi^{\prime}$ on $X^{\prime}$. Then $\alpha:(X, \xi) \rightarrow\left(X^{\prime}, \xi^{\prime}\right)$ is a uniform map. Now Lemma 2.3(4) implies that the identity map $\left(X^{\prime}, \tau^{\prime}\right) \rightarrow\left(X^{\prime}, \xi^{\prime}\right)$ is fragmented. This means in view of Definition 2.7 that $F^{\prime}$ is a fragmented family. 
Lemma 2.9. (1) Suppose $F$ is a compact space, $X$ is Čech-complete, $M$ is a metrizable space and we are given a separately continuous map $w: X \times F \rightarrow$ $M$. Then the naturally associated family

$$
\tilde{F}:=\{\tilde{f}: X \rightarrow M\}_{f \in F}
$$

is fragmented, where $\tilde{f}(x)=w(x, f)$.

(2) Suppose $F$ is a compact and metrizable space, $X$ is Polish and $M$ is separable and metrizable. Assume we are given a map $w: X \times F \rightarrow M$ such that every $\tilde{x}: F \rightarrow M, f \mapsto w(x, f)$ is continuous and $y: X \rightarrow M$ is continuous at every $\tilde{y} \in Y$ for some dense subset $Y$ of $F$. Then the family $\tilde{F}$ is barely continuous (hence, fragmented).

Proof. (1): Use Namioka's joint continuity theorem 32.

(2): Since every $\tilde{x}: F \rightarrow M$ is continuous, the natural map $j: X \rightarrow C(F, M)$, $j(x)=\tilde{x}$ is well defined. For every closed nonempty subset $A \subset X$ the induced map $\left.j\right|_{A}: A \rightarrow C(F, M)$ has a point of continuity by virtue of [16, Proposition 2.4], where $C(F, M)$ carries the sup-metric. Hence, $\tilde{F}_{A}=\left\{\tilde{f}\left\lceil_{A}: A \rightarrow M\right\}_{f \in F}\right.$ is equicontinuous at some point $a \in A$. This means, by Definition 2.7(2), that the family $\tilde{F}$ is barely continuous.

Definition 2.10. We say that a family of functions $F=\{f:(X, \tau) \rightarrow(Y, \mu)\}$ is eventually fragmented if every sequence in $F$ has a subsequence which is a fragmented family on $X$.

Example 2.11. Let $V$ be a Banach space. Then we can treat the unit ball $B_{V}$ as a family of functions on the weak ${ }^{*}$ compact space $B_{V^{*}}$.

(1) $B_{V}$ is a fragmented family of functions on $B_{V^{*}}$ if and only if $V$ is Asplund. This fact easily follows from the well-known characterization of Asplund spaces: $V$ is Asplund iff $B_{V^{*}}$ is (weak* ${ }^{*}$, norm)-fragmented (Remark 2.2(4)).

(2) $B_{V}$ is an eventually fragmented family of functions on $B_{V^{*}}$ if and only if $V$ is a Rosenthal Banach space (see Proposition 4.12).

\section{Dynamical BaCkground: $G$-Spaces And Banach Representations}

By a $G$-space (or a $G$-system) $X$ we mean a continuous action of a topological group $G$ on a topological space $X$. Sometimes we write it as $(G, X)$. The Banach algebra (under the supremum norm) of all continuous real-valued bounded functions on a topological space $X$ will be denoted by $C(X)$. The material in this section is mostly well known. For more details and undefined concepts, see for example [13, 12, 14].

3.1. Cyclic $G$-spaces. A function $f \in C(X)$ on a $G$-space $X$ comes from a compact $G$-space $Y$ if there exist a $G$-compactification $\nu: X \rightarrow Y$ (so, $\nu$ is onto if $X$ is compact) and a function $f_{0} \in C(Y)$ such that $f=f_{0} \circ \nu$. Then necessarily, $f$ is right uniformly continuous (notation: $f \in \operatorname{RUC}(X)$ ); that is, the orbit map $f: G \rightarrow C(X), g \mapsto f g$ is norm continuous. For every $G$-space $X$ a function $f: X \rightarrow \mathbb{R}$ lies in $\operatorname{RUC}(X)$ iff it comes from a compact $G$-space $Y$. Among all possible $G$-compactifications $\nu: X \rightarrow Y$ of a $G$-space $X$ such that $f$ comes from $(\nu, Y)$ there exists the smallest one. One may define it by the smallest closed unital $G$-subalgebra $\mathcal{A}_{f}$ of $\operatorname{RUC}(X)$ generated by the orbit $f G$ of $f$ in $\operatorname{RUC}(X)$. 
Denote by $X_{f}$ the Gelfand space of the algebra $\mathcal{A}_{f}$. Then the corresponding $G$ compactification $\alpha_{f}: X \rightarrow Y:=X_{f}$ is the required one. We call $X_{f}$ the cyclic $G$-system of $f$. The function $f$ comes from $X_{f}$. There exists a continuous function $f_{0}: X_{f} \rightarrow \mathbb{R}$ such that $f=f_{0} \circ \alpha_{f}$ and furthermore the family $f_{0} G$ separates points of $X_{f}$.

3.2. Enveloping semigroups. The enveloping (or Ellis) semigroup $E=E(G, X)$ $=E(X)$ of a compact dynamical $G$-system $X$ is defined as the closure in $X^{X}$ (with its compact pointwise convergence topology) of the set $\breve{G}=\{\breve{g}: X \rightarrow X\}_{g \in G}$ of translations. With the operation of composition of maps, $E(X)$ is a right topological semigroup. Moreover, the map

$$
j=j_{X}: G \rightarrow E(X), g \mapsto \breve{g}
$$

is a right topological semigroup compactification of $G$. The compact space $E(X)$ becomes a $G$-space with respect to the natural action

$$
G \times E(X) \rightarrow E(X), \quad(g p)(x)=g p(x) .
$$

Let $E=E(X)$ be the enveloping semigroup of a compact $G$-system $X$. For every $f \in C(X)$ define

$$
E^{f}:=\{f \circ p: X \rightarrow \mathbb{R}\}_{p \in E} .
$$

Then $E^{f}$ is a pointwise compact subset of $\mathbb{R}^{X}$, being a continuous image of $E$ under the map $q_{f}: E \rightarrow E^{f}, \quad p \mapsto f \circ p$. The orbit $f G$ of $f$ is a dense subset of $E^{f}$. It follows that $E^{f}=\operatorname{cls}_{p}(f G)$.

3.3. Banach representations of dynamical systems. Let $V$ be a Banach space. Denote by Iso $(V)$ the topological group of all linear isometries of $V$ onto itself, equipped with the pointwise convergence topology.

Definition 3.1 ([30]). Let $X$ be a $G$-space. A continuous representation of $(G, X)$ on a Banach space $V$ is a pair

$$
h: G \rightarrow \operatorname{Iso}(V), \quad \alpha: X \rightarrow V^{*},
$$

where $h: G \rightarrow$ Iso $(V)$ is a continuous co-homomorphism and $\alpha: X \rightarrow V^{*}$ is a weak* continuous bounded $G$-map with respect to the dual action $G \times V^{*} \rightarrow$ $V^{*},(g \varphi)(v):=\varphi(h(g)(v))$. We say that a continuous representation $(h, \alpha)$ is faithful when $\alpha$ is a topological embedding.

Every compact $G$-space $X$ admits a canonical faithful representation on the Banach space $C(X)$. A natural question is to characterize dynamical systems according to their representability on nice Banach spaces.

3.3.1. Reflexive representations and WAP systems. A compact dynamical system $(G, X)$ is weakly almost periodic (WAP) if $C(X)=W A P(X)$. As usual a continuous function $f: X \rightarrow \mathbb{R}$ is WAP if the weak closure of the orbit $f G$ is weakly compact in $C(X)$. A compact $G$-space $X$ is WAP iff every element $p \in E(X)$ is a continuous self-map of $X$ (see Ellis and Nerurkar [7]).

Fact 3.2 (See [30]). Let $X$ be a compact metric $G$-space. The following conditions are equivalent:

(1) $(G, X)$ is weakly almost periodic (WAP).

(2) $(G, X)$ is reflexively representable (that is, admits a faithful representation on a reflexive Banach space). 
3.3.2. Asplund representations, $R N$ and HNS systems. A dynamical system is Radon-Nikodým (RN) if it admits a faithful representation on an Asplund Banach space [30, 13. If $G=\{1\}$, we get the class of Radon-Nikodým compact spaces in the sense of Namioka [33].

We recall the concept of nonsensitivity (see for instance [17, 1, 30, 13, 15] and the references therein). Let $d$ be a compatible metric on a compact $G$-system $X$. We say that $(G, X)$ is nonsensitive if for every $\varepsilon>0$ there exists a nonempty open set $O \subset X$ such that for every $g \in G$ the set $g O$ has $d$-diameter $<\varepsilon$. $(G, X)$ is hereditarily nonsensitive (HNS) if all its closed $G$-subspaces are nonsensitive.

For a nonmetrizable version of HNS in terms of uniform structures and some related properties, we refer to [13].

Fact 3.3 (See [13] and [16]). Let $X$ be a compact metric $G$-space. The following conditions are equivalent:

(1) $(G, X)$ is hereditarily nonsensitive (HNS).

(2) $(G, X)$ is $\mathrm{RN}$, that is, admits a faithful representation on an Asplund Banach space.

(3) The enveloping semigroup $E(X)$ is metrizable.

Fact 3.4 ([13]). Let $X$ be a compact $G$-space. The following conditions are equivalent:

(1) $(G, X)$ is HNS.

(2) $(G, X)$ is $\mathrm{RN}$-approximable, that is, admits sufficiently many representations on Asplund Banach spaces.

(3) $E(X)=\{p: X \rightarrow X\}_{p \in E(X)}$ is a fragmented family.

(4) $\breve{G}=\{\breve{g}: X \rightarrow X\}_{g \in G}$ is a fragmented family.

\section{BANACH SPACE BACKGROUND AND THE DYNAMiCAL BFT DiChOtomy}

4.1. Bourgain-Fremlin-Talagrand theorems. Recall that a topological space $K$ is a Rosenthal compactum [18] if it is homeomorphic to a pointwise compact subset of the space $\mathcal{B}_{1}(X)$ of functions of the first Baire class on a Polish space $X$. All metric compact spaces are Rosenthal. An example of a separable nonmetrizable Rosenthal compact is the Helly compact 8 of all (not only strictly) increasing selfmaps of $[0,1]$ in the pointwise topology. Another is the "two arrows" space [8] of Alexandroff and Urysohn. Recall that a topological space $K$ is Fréchet (or, FréchetUrysohn [8]) if for every $A \subset K$ and every $x \in \operatorname{cls}(A)$ there exists a sequence of elements of $A$ which converges to $x$.

The following theorem is due to Bourgain-Fremlin-Talagrand [2, Theorem 3F], generalizing a result of Rosenthal. The second assertion (BFT dichotomy) is presented as in the book of Todorčević [43] (see Proposition 1 of Section 13).

Fact 4.1. (1) Every Rosenthal compact space $K$ is Fréchet.

(2) (BFT dichotomy) Let $X$ be a Polish space and let $\left\{f_{n}\right\}_{n=1}^{\infty} \subset C(X)$ be a pointwise bounded sequence of bounded functions. Let $K$ be the pointwise closure of $\left\{f_{n}\right\}_{n=1}^{\infty}$ in $\mathbb{R}^{X}$. Then either $K \subset \mathcal{B}_{1}(X)$ (i.e., $K$ is Rosenthal compact) or $K$ contains a homeomorphic copy of $\beta \mathbb{N}$.

Clearly, $\beta \mathbb{N}$, the Stone-Čech compactification of the natural numbers $\mathbb{N}$, is not Fréchet, and hence it is not a Rosenthal compact space. 
Definition 4.2. Let $X$ be a topological space. We say that a subset $F \subset C(X)$ is a Rosenthal family (for $X$ ) if $F$ is norm bounded and the pointwise closure $\operatorname{cls}_{p}(F)$ of $F$ in $\mathbb{R}^{X}$ consists of fragmented maps, that is,

$$
\operatorname{cls}_{p}(F) \subset \mathcal{F}(X) .
$$

In the following result we combine two theorems from Talagrand's book [42]. Here we reformulate assertion (3) in terms of $\mathcal{F}(X)$ using the equality $\mathcal{F}(X)=$ $B_{r}^{\prime}(X)$ (Corollary 2.6, 1 ).

Fact 4.3 (Talagrand [42]). Let $X$ be a compact space and $F \subset C(X)$ a bounded subset. The following conditions are equivalent:

(1) $F$ does not contain a subsequence equivalent to the unit basis of $l_{1}$.

(2) Each sequence in $F$ has a pointwise convergent subsequence in $\mathbb{R}^{X}$ (i.e., $F$ is sequentially precompact in $\left.\mathbb{R}^{X}\right)$.

(3) $\operatorname{cls}_{p}(F) \subset \mathcal{F}(X)$ (i.e., $F$ is a Rosenthal family for $X$ ).

(4) Every sequence in $F$ has a weak-Cauchy subsequence.

(5) Every countable subfamily $S$ of $F$ is a Rosenthal family for $X$.

Proof. The equivalence of (1), (2) and (3) is a part of [42, Theorem 14.1.7]. The equivalence of (2) and (4) is a part of [42, Theorem 7.3.1]. The equivalence of the assertions (1), (2), (3) and (4) implies now that each of them is equivalent also to (5).

Let $F \subset C(X)$ be a norm bounded subset. Then the pointwise $\operatorname{closure~} \operatorname{cls}_{p}(F)$ in $\mathbb{R}^{X}$ is compact. The following lemma examines four natural conditions expressing "smallness" of $F$ (compare Proposition [5.9).

Lemma 4.4. Let $F \subset C(X)$ be a norm bounded family, where $X$ is a Polish space and cls $_{p}(F)$ is the (necessarily compact) pointwise closure of $F$ in $\mathbb{R}^{X}$. Consider the following conditions:

(a) $\operatorname{cls}_{p}(F) \subset C(X)$.

(b) $c l s_{p}(F)$ is a metrizable subspace in $\mathbb{R}^{X}$.

(c) $F$ is a fragmented (equivalently, barely continuous) family of functions on $X$.

(d) $\operatorname{cls}_{p}(F) \subset \mathcal{B}_{1}(X)$.

Then always $(a) \Longrightarrow(b) \Longleftrightarrow(c) \Longrightarrow(d)$.

Proof. (a) $\Rightarrow($ b): Let $Y$ be a dense countable subset of $X$. Since every function $\phi \in \operatorname{cls}_{p}(F)$ is continuous we get that the natural continuous projection $\mathbb{R}^{X} \rightarrow \mathbb{R}^{Y}$ induces an injection on $\operatorname{cls}_{p}(F)$. Since $\operatorname{cls}_{p}(F)$ is compact we get its homeomorphic embedding into the second countable space $\mathbb{R}^{Y}$.

(b) $\Rightarrow$ (c): Apply Lemma 2.9(2) to the evaluation map $X \times \operatorname{cls}_{p}(F) \rightarrow \mathbb{R}$. It follows that $\operatorname{cls}_{p}(F)$ and hence $F$ are fragmented families.

(c) $\Rightarrow(\mathrm{b})$ : The family $F$ is fragmented means by Definition 2.7 that the natural map $X \rightarrow \mathbb{R}^{F}$ is fragmented, where $\mathbb{R}^{F}$ carries the uniformity of uniform convergence. Then the image of $X$ is separable as it follows by [13, Lemma 6.5]. Now [33, Theorem 4.1] implies that the pointwise $\operatorname{closure~} \operatorname{cls}_{p}(F)$ of $F$ in $\mathbb{R}^{X}$ is metrizable.

$(c) \Rightarrow(d)$ : Since $F$ is a fragmented family its pointwise closure $\operatorname{cls}_{p}(F)$ is again a fragmented family (Lemma 2.8.1). In particular, every member $\phi \in \operatorname{cls}_{p}(F)$ is a fragmented map on $X$. Since $X$ is Polish this means by Corollary 2.6 that $\phi \in \mathcal{B}_{1}(X)$. 
Lemma 4.5. Let $q: X_{1} \rightarrow X_{2}$ be a map between topological spaces. Then

(1) The natural map $\gamma: \mathbb{R}^{X_{2}} \rightarrow \mathbb{R}^{X_{1}}, \gamma(\phi)=\phi \circ q$ is pointwise continuous.

(2) If $q: X_{1} \rightarrow X_{2}$ is onto, then $\gamma$ is injective.

(3) Let $q: X_{1} \rightarrow X_{2}$ be a continuous onto map between compact spaces, $F_{2} \subset$ $C\left(X_{2}\right)$ and $F_{1} \subset C\left(X_{1}\right)$ be norm bounded subsets such that $F_{1}=F_{2} \circ q$. Then

(a) $F_{1}$ is a Rosenthal family for $X_{1}$ if and only if $F_{2}$ is a Rosenthal family for $X_{2}$.

(b) $\gamma$ induces a homeomorphism between the compact spaces $c s_{p}\left(F_{2}\right)$ and $c l s_{p}\left(F_{1}\right)$.

Proof. Claims (1) and (2) are trivial.

(3)(a): By the continuity of $\gamma$ we get $\gamma\left(F_{2}\right) \subset \gamma\left(\operatorname{cls}_{p}\left(F_{2}\right)\right) \subset \operatorname{cls}_{p}\left(\gamma\left(F_{2}\right)\right)$. Since $F_{2}$ is bounded the set $\operatorname{cls}_{p}\left(F_{2}\right)$ is compact in $\mathbb{R}^{X_{2}}$. Then $\gamma\left(\operatorname{cls}_{p}\left(F_{2}\right)\right)=\operatorname{cls}_{p}\left(\gamma\left(F_{2}\right)\right)$. On the other hand, $\gamma\left(F_{2}\right)=F_{2} \circ q=F_{1}$. Therefore, $\operatorname{cls}_{p}\left(F_{2}\right) \circ q=\operatorname{cls}_{p}\left(F_{1}\right)$. Now apply Lemma $2.3(5)$.

(3)(b): Combine the assertions (1) and (2), taking into account that $\gamma\left(\operatorname{cls}_{p}\left(F_{2}\right)\right)$ $=\operatorname{cls}_{p}\left(\gamma\left(F_{2}\right)\right)=\operatorname{cls}_{p}\left(F_{1}\right)$.

Proposition 4.6. Let $X$ be a compact space and $F$ a bounded subset of $C(X)$. The following conditions are equivalent:

(1) $F$ is a Rosenthal family for $X$.

(2) Every sequence in $F$ has a subsequence which is an eventually fragmented family on $X$ (i.e. $F$ is a subfragmented family of maps on $X$ ).

If $X$ is metrizable, then each of these conditions is equivalent to the following:

(3) The pointwise closure cls $p_{p}(F)$ of $F$ in $\mathbb{R}^{X}$ is a subset of $\mathcal{B}_{1}(X)$.

Proof. (1) $\Rightarrow(2)$ : Let $S$ be a sequence in $F$. By the implication (3) $\Rightarrow$ (2) from Fact 4.3 we can choose a pointwise convergent subsequence $\left\{f_{n}\right\}_{n \in \mathbb{N}}$ of $S$ with $\phi=\lim f_{n}$. Denote by $K$ the compact metrizable subset $\left\{f_{n}\right\}_{n \in \mathbb{N}} \cup\{\phi\}$ in $\mathbb{R}^{X}$. Define the pointwise continuous map

$$
q: X \rightarrow \mathbb{R}^{S}, q(x)(f)=f(x) \quad \forall f \in S
$$

and denote by $X^{\prime}$ the subspace $q(X) \subset \mathbb{R}^{S}$. Clearly, $X^{\prime}$ is pointwise compact because $S$ is norm bounded. Furthermore, $X^{\prime}$ is metrizable since $S$ is countable. For every $f \in S$ we have the uniquely defined continuous map $f^{\prime}: X^{\prime} \rightarrow \mathbb{R}$, $f^{\prime}(q(x))=f(x)$ such that $f=f^{\prime} \circ q=\gamma\left(f^{\prime}\right)$. By Lemma 4.5(3)(b), $\gamma: \mathbb{R}^{X} \rightarrow \mathbb{R}^{X^{\prime}}$ induces a homeomorphism between the compact spaces $\operatorname{cls}_{p}\left(A^{\prime}\right) \rightarrow \operatorname{cls}_{p}(A)$, where $A:=\left\{f_{n}\right\}_{n \in \mathbb{N}}$ and $A^{\prime}:=\left\{f_{n}^{\prime}\right\}_{n \in \mathbb{N}}$. Therefore, there exists $\psi \in X^{\prime}$ with $\gamma(\psi)=\phi$ such that $\gamma$ induces a homeomorphism $K^{\prime} \rightarrow K$, where $K^{\prime}:=\left\{f_{n}^{\prime}\right\}_{n \in \mathbb{N}} \cup\{\psi\}$. Consider the evaluation map $X^{\prime} \times K^{\prime} \rightarrow \mathbb{R}$. Then we can apply Lemma 2.9 (2), which implies that $K^{\prime}$, and hence also its subfamily $A^{\prime}$, are fragmented families of maps on $X^{\prime}$. Now Lemma 2.8 (2) implies that $A$ is a fragmented family of maps on $X$.

$(2) \Rightarrow(1)$ : We have to show that $F$ is a Rosenthal family for $X$. By Fact 4.3 it is equivalent to checking that every sequence $S$ in $F$ has a pointwise convergent subsequence in $\mathbb{R}^{X}$. By our assumption there exists a subsequence of $S$ which is a fragmented family of functions on $X$. So without restriction of generality we may assume that $S$ itself is a fragmented family. As in the proof above consider the quotient $q: X \rightarrow X^{\prime} \subset \mathbb{R}^{S}$. Then the family $S^{\prime}:=\left\{f^{\prime}: X^{\prime} \rightarrow \mathbb{R}\right\}_{f \in S}$ is a fragmented 
family by Lemma 2.8(2). Now by Lemma 4.4 the pointwise closure $\operatorname{cls}_{p}\left(S^{\prime}\right)$ is a (compact) metrizable subspace in $\mathbb{R}^{X^{\prime}}$. Therefore there exists a convergent subsequence of $S^{\prime}$ (in $\operatorname{cls}_{p}\left(S^{\prime}\right) \subset \mathbb{R}^{X^{\prime}}$ ). By Lemma 4.5(3)(b), $\gamma: \mathbb{R}^{X} \rightarrow \mathbb{R}^{X^{\prime}}$ induces a homeomorphism between the compact $\operatorname{spaces} \operatorname{cls}_{p}(S)$ and $\operatorname{cls}_{p}\left(S^{\prime}\right)$. Hence there exists a convergent subsequence of $S\left(\right.$ in $\mathbb{R}^{X}$ ), as desired.

$(1) \Leftrightarrow(3)$ (For metrizable $X)$ : Since $X$ is compact metrizable we have $\mathcal{F}(X)=$ $\mathcal{B}_{1}(X)$ by Corollary 2.6 (2).

Let $X=\{0,1\}^{\mathbb{N}}$ be the Cantor cube and $F:=\left\{p_{n}\right\}_{n \in \mathbb{N}}$ the family of all projection mappings (with $p_{n}(x)=x(n)$ ). It is well known that the pointwise closure of $F$ in $\mathbb{R}^{X}$ is homeomorphic to $\beta \mathbb{N}$ (see for instance [43, p. 4]). By Fact 4.1 it follows that $F$ is not a Rosenthal family for $X=\{0,1\}^{\mathbb{N}}$.

\subsection{Banach spaces not containing $l_{1}$.}

Definition 4.7. Let us say that a Banach space $V$ is Rosenthal if it does not contain an isomorphic copy of $l_{1}$.

Clearly the class of Asplund spaces (see Remarks 2.2(4)) is a subclass of the class of Rosenthal spaces. The difference between these two classes can be illustrated in terms of fragmentability. Compare the last two items of Remarks 2.2 and Proposition 4.12

Recall the following famous result of Rosenthal.

Fact 4.8 (Rosenthal [38]). Let $V$ be a Banach space. The following conditions are equivalent:

(1) $V$ is a Rosenthal Banach space.

(2) Every bounded sequence in $V$ has a weak-Cauchy subsequence.

Every Banach space $V$ can be treated as a natural subspace of the Banach space $C\left(B_{V^{*}}\right)$, where $B_{V^{*}}$ is the weak ${ }^{*}$ compact unit ball of $V^{*}$. Furthermore, the corresponding weak topology on $V$ coincides with the weak topology inherited from $C\left(B_{V^{*}}\right)$. Therefore taking into account Fact 4.3 and Definition 4.2 we get the following reformulation of Fact 4.8 .

Lemma 4.9. Let $V$ be a Banach space. The following conditions are equivalent:

(1) $V$ is a Rosenthal Banach space.

(2) The unit ball $B_{V}$ of $V$ is a Rosenthal family for the weak* compact unit ball $B_{V^{*}}$ of $V^{*}$.

For the separable case, we have the following theorem.

Fact 4.10 ([35, [39, p. 374] and [40, Theorem 3]). Let $V$ be a separable Banach space. The following conditions are equivalent:

(1) $V$ is a Rosenthal Banach space.

(2) $\operatorname{card}\left(V^{* *}\right)=\operatorname{card}(V)$

(3) Every $v^{* *} \in V^{* *}$ is a Baire 1 function when restricted to $B_{V^{*}}$.

Thus a separable Banach space $V$ does not contain an isomorphic copy of $l_{1}$ if and only if every element $x^{* *} \in V^{* *}$ is Baire 1 when restricted to the unit ball $B_{V^{*}}$ with its weak* topology $\sigma\left(V^{*}, V\right)$. This classical result of Odell and Rosenthal was generalized in [41]. 
Let $A$ be a weak* compact subset of a dual Banach space $V^{*}$. Following [37] we say that $A$ has the scalar point of continuity property if for each weak* compact subset $M$ of $A$ and every $x^{* *} \in V^{* *}$, the restriction $\left.x^{* *}\right|_{M}$ of $x^{* *}$ to $M$ has a point of continuity.

Fact 4.11 (E. Saab and P. Saab [4]). A Banach space $V$ (separable or not) is Rosenthal if and only if $B_{V^{*}}$ has the scalar point of continuity property.

The following result gives three characterizations of Rosenthal spaces in terms of fragmentability.

Proposition 4.12. Let $V$ be a Banach space. The following conditions are equivalent:

(1) $V$ is a Rosenthal Banach space.

(2) Each $x^{* *} \in V^{* *}$ is a fragmented map when restricted to the weak* compact ball $B_{V^{*}}$.

(3) Every bounded subset $A$ of the dual $V^{*}$ is $\left(\tau_{w^{*}}, \mu_{w}\right)$-fragmented (that is, $i d_{A}:\left(A, \tau_{w^{*}}\right) \rightarrow\left(A, \mu_{w}\right)$ is fragmented $)$, where $\tau_{w^{*}}$ is the weak $k^{*}$ topology and $\mu_{w}$ is the weak uniformity on $A$.

(4) $B_{V}$ is an eventually fragmented family of functions on $\left(B_{V^{*}}, w^{*}\right)$.

Proof. By Corollary 2.6(1), $\mathcal{F}(X)=B_{r}^{\prime}(X)$ for $X=B_{V^{*}}$. Now Fact 4.11 yields the equivalence $(1) \Leftrightarrow(2)$.

$(1) \Leftrightarrow(3)$ : This follows by Fact 4.11 and Lemma 2.5(1), taking into account Remark 2.2(2).

For $(1) \Leftrightarrow(4)$ use Proposition 4.6 and Lemma 4.9 .

Remark 4.13. (1) The equivalence of (1) and (2) in Proposition 4.12 is indeed a natural generalization of the Odell-Rosenthal result [35] because for compact metrizable $X$ we have $\mathcal{B}_{1}(X)=\mathcal{F}(X)$ (Corollary 2.6 (2)) and the weak* compact ball $B_{V^{*}}$ is metrizable for separable $V$.

(2) Let $V$ be a Banach space and $A$ a weak* compact absolutely convex subset of $V^{*}$. Then by [37, Theorem 9], $A$ has the scalar point of continuity property if and only if $A$ is a weak Radon-Nikodým subset (WRN for short). We refer to [3, 41, 37] for exact definitions and additional information about WRN subsets. See also Theorem 6.5 below about WRN compact spaces.

4.3. Convex hulls. The following result is proved in Bourgain-Fremlin-Talagrand [2].

Fact 4.14 ([2, Theorem 5E]). Let $X$ be a complete metric space, $A \subset \mathcal{B}_{1}(X)$ a pointwise compact uniformly bounded set. Then its convex hull $\operatorname{co}(A)$ is relatively compact in $\mathcal{B}_{1}(X)$ (equivalently, $\operatorname{cls}_{p}(\operatorname{co}(A)) \subset \mathcal{B}_{1}(X)$ ).

For Rosenthal families we get the following result.

Proposition 4.15. Let $F$ be a Rosenthal family for a compact space $X$. Then its convex hull $\mathrm{co}(F)$ is also a Rosenthal family for $X$.

Proof. First case: For a compact metrizable $X$ combine Fact 4.14 and Proposition 4.6.

Second case: For a general compact space $X$, by Fact 4.3 we have only to examine sequences. That is, it is enough to show that every countable subset $M$ of $c o(F)$ is a Rosenthal family. There exists a countable subset $S \subset F$ such that $M \subset \operatorname{co}(S)$. 
As in the proof of Proposition 4.6 consider the quotient $q: X \rightarrow X^{\prime} \subset \mathbb{R}^{S}$ induced by the collection $S$. Then every $f \in S$ induces a continuous map $f^{\prime}: X^{\prime} \rightarrow \mathbb{R}$ such that $f=f^{\prime} \circ q=\gamma\left(f^{\prime}\right)$.

By our assumption, $S$ is a Rosenthal family for $X$. Then $S^{\prime}:=\left\{f^{\prime}: f \in S\right\}$ is a Rosenthal family for $X^{\prime}$ (use Lemma 4.5(3)). Since $X^{\prime}$ is metrizable we can apply the first case and deduce that the convex hull $\operatorname{co}\left(S^{\prime}\right)$ is a Rosenthal family for $X^{\prime}$. The map

$$
\gamma: \mathbb{R}^{X^{\prime}} \rightarrow \mathbb{R}^{X}, \gamma(\phi)=\phi \circ q
$$

(see Lemma 4.5) is linear and $\gamma\left(S^{\prime}\right)=S$. Therefore, $\gamma\left(\operatorname{co}\left(S^{\prime}\right)\right)=\operatorname{co}(S)$. It follows, by Lemma $4.5(3)$, that the collection $c o(S)$, and hence its subcollection $M$, is a Rosenthal family for $X$.

4.4. The natural affine extension map $T: b \mathcal{B}_{1}(X) \rightarrow b \mathcal{B}_{1}\left(B^{*}\right)$. For every compact metric space $X$, denote by $b \mathcal{B}_{1}(X)$ the collection of bounded Baire 1 realvalued functions on $X$. That is,

$$
b \mathcal{B}_{1}(X)=\mathcal{B}_{1}(X) \cap l_{\infty}(X)
$$

Then $b \mathcal{B}_{1}(X)$ is a topological subspace of $\mathcal{B}_{1}(X)$ with respect to the pointwise topology (inherited from $\mathbb{R}^{X}$ ). One can define a natural injective map

$$
T: b \mathcal{B}_{1}(X) \rightarrow b \mathcal{B}_{1}\left(B^{*}\right),
$$

where $B^{*}$, as before, is the weak* compact unit ball of $C(X)^{*}$. We will use the Riesz Representation Theorem and Lebesgue's Dominated Convergence Theorem.

Each $f \in b \mathcal{B}_{1}(X)$ is universally measurable for every compact metric space $X$ (see for example [2, Proposition $1 \mathrm{~F}]$ ). That is, for every measure $\mu \in B^{*}$ we can define

$$
(T f)(\mu):=\int f d \mu .
$$

This map is well defined. Indeed, first note that when $f \in C(X), T(f)=i(f)$, where

$$
i: C(X) \hookrightarrow C\left(B^{*}\right), \quad i(f)(\mu):=\langle f, \mu\rangle=\int f d \mu
$$

is the canonical isometric inclusion of the corresponding Banach spaces and

$$
\langle\cdot, \cdot\rangle: C(X) \times C(X)^{*} \rightarrow \mathbb{R}
$$

is the canonical bilinear mapping. Now if $f \in b \mathcal{B}_{1}(X)$, then $f$ is a pointwise limit of a sequence of continuous functions $h_{n} \in C(X)$ (Lemma 2.4(2)). Since $f: X \rightarrow \mathbb{R}$ is a bounded function we can assume in addition that the sequence $h_{n}$ is uniformly bounded. By Lebesgue's Convergence Theorem it follows that $T(f)$ is a pointwise limit of the sequence $T\left(h_{n}\right)=i\left(h_{n}\right), n \in \mathbb{N}$. Since every $i\left(h_{n}\right) \in C\left(B^{*}\right)$ we conclude by Lemma 2.4 (2) that $T(f) \in \mathcal{B}_{1}\left(B^{*}\right)$. The sequence $i\left(h_{n}\right)$ is uniformly bounded in $C\left(B^{*}\right)$; hence $T(f)$ is a bounded function. This means that $T(f) \in b \mathcal{B}_{1}\left(B^{*}\right)$.

The map $T$ is injective because $T(f)\left(\delta_{x}\right)=f(x)$ for every point mass $\delta_{x} \in B^{*}$ $(x \in X)$.

Remark 4.16. Each $T(f)$ for $f \in b \mathcal{B}_{1}(X)$ can be treated as an element of the second dual $C(X)^{* *}$ of $C(X)$. Moreover the pointwise topology of $\mathcal{B}_{1}\left(B^{*}\right)$ and the weak*-topology on $C(X)^{* *}$ agree on $T\left(b \mathcal{B}_{1}(X)\right)$. 
Lemma 4.17. Let $X$ be a compact metric space. For every uniformly bounded subset $A \subset b \mathcal{B}_{1}(X)$ the restriction $\left.T\right|_{A}$ of the natural injective map

$$
T: b \mathcal{B}_{1}(X) \rightarrow b \mathcal{B}_{1}\left(B^{*}\right) \cap C(X)^{* *}
$$

on $A$ is sequentially continuous. Furthermore, $T(A)$ is also uniformly bounded.

Proof. Lebesgue's Convergence Theorem implies that $T$ is sequentially continuous. The boundedness of $T(A)$ is easy.

Proposition 4.18. If $F \subset C(X)$ is a Rosenthal family for a compact metric space $X$, then the restriction of $T$ on $\operatorname{cls}_{p}(F)$ induces a homeomorphism

$$
c l s_{p}(F) \rightarrow \operatorname{cls}_{p}(T(F)) \subset b \mathcal{B}_{1}\left(B^{*}\right) \cap C(X)^{* *} .
$$

Proof. As $F$ is a Rosenthal family for $X$ its pointwise $\operatorname{closure~} \operatorname{cls}_{p}(F)$ is a compact subset of $\mathcal{B}_{1}(X)$. Moreover, $\operatorname{cls}_{p}(F)$ is a uniformly bounded subset of $b \mathcal{B}_{1}(X)$ because $F$ is bounded (Definition 4.2). In view of Lemma 4.17 the restricted map $T: \operatorname{cls}_{p}(F) \rightarrow b \mathcal{B}_{1}\left(B^{*}\right)$ is sequentially continuous. By the Bourgain-FremlinTalagrand theorem, Fact 4.1(1), we know that $\operatorname{cls}_{p}(F)$ is Fréchet. For a Fréchet space a sequentially continuous map is continuous and we conclude that the map $T: \operatorname{cls}_{p}(F) \rightarrow b \mathcal{B}_{1}\left(B^{*}\right)$ is a continuous injection, and therefore a homeomorphism, of $\operatorname{cls}_{p}(F)$ onto its image in $b \mathcal{B}_{1}\left(B^{*}\right)$.

Proposition 4.19. Let $X$ be a compact space and $F \subset C(X)$. The following conditions are equivalent:

(1) $F$ is a Rosenthal family for $X$.

(2) $F$ is a Rosenthal family for $B^{*}$.

Proof. We use Fact 4.3 which depends on sequences only. Since $F$ is a Rosenthal family for $X$, by Fact 4.3 the set $F$ is sequentially precompact in $\mathbb{R}^{X}$ (that is, every sequence in $F$ has a subsequence which converges in $\mathbb{R}^{X}$ ). Since $F$ is bounded we can apply Lebesgue's Dominated Convergence Theorem, which implies that $F$ is sequentially precompact also in $\mathbb{R}^{B^{*}}$. Thus $\operatorname{cls}_{p}(F) \subset \mathcal{F}\left(B^{*}\right)$ by Fact 4.3 . Hence $F$ is a Rosenthal family for $B^{*}$.

\section{TAme Dynamical Systems}

A compact metric dynamical $G$-system $X$ is called tame [10] if in the dynamical BFT-dichotomy (Fact 1.2) the first alternative occurs; i.e., $E(X)$ is Rosenthal compact (see also Proposition 6.15 below).

Fact 5.1 ([16]). A compact metric dynamical $G$-system $X$ is tame if and only if every element of $E(X)$ is a Baire 1 function (equivalently, fragmented) from $X$ to itself.

This result suggests the following general definition.

Definition 5.2. Let $X$ be a (not necessarily metrizable) compact $G$-space. We say that $X$ is tame if for every element $p \in E(X)$ the function $p: X \rightarrow X$ is fragmented (that is, if $E(X) \subset \mathcal{F}(X, X)$ ).

We will see later that this class is the same as the class of all regular systems in the sense of Köhler [26]. In particular this gives an enveloping semigroup characterization of regular systems. 
Lemma 5.3. Every compact HNS dynamical G-system is tame.

Proof. $E(X)=\{p: X \rightarrow X\}_{p \in E(X)}$ is a fragmented family when $X$ is HNS by Fact 3.4. In particular we get $E(X) \subset \mathcal{F}(X, X)$.

Roughly speaking the difference between HNS and tame systems is the difference between "fragmented families" and "families which consist of fragmented maps" (see Facts 3.4 and 5.1).

Lemma 5.4. For every $G$ the class of tame $G$-systems is closed under subsystems, arbitrary products and factors.

Proof. The case of subsystems is trivial because the fragmentability of maps is a hereditary property. The cases of products and factors can both be proved using Lemma 2.3

For factors: Let $\alpha: X \rightarrow Y$ be a $G$-factor. By [6, Prop. 3.8] there exists a (unique) continuous onto semigroup homomorphism $Q: E(X) \rightarrow E(Y)$ such that $\alpha \circ p=Q(p) \circ \alpha$ for every $p \in E(X)$. Since $Q$ is onto for every $p_{Y} \in E(Y)$ there exists $p_{X} \in E(X)$ such that the following diagram commutes:

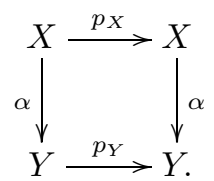

Then $p_{X} \in \mathcal{F}(X, X)$ because $(G, X)$ is tame. By Lemma 2.3(4) we obtain that $p_{Y} \in \mathcal{F}(Y, Y)$. This shows that $(G, Y)$ is tame.

For products: Let $X:=\prod_{i \in I} X_{i}$ be a $G$-product of compact tame $G$-spaces $X_{i}$ with canonical $G$-projections $\alpha_{i}: X \rightarrow X_{i}$. For every $p \in E(X)$ and every index $i$ we have the following commutative diagram:

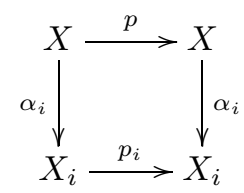

for some $p_{i} \in E\left(X_{i}\right)$. By our assumption, $p_{i} \in \mathcal{F}\left(X_{i}, X_{i}\right)$ because $\left(G, X_{i}\right)$ is tame. Hence each $p_{i} \circ \alpha_{i}$ belongs to $\mathcal{F}(X, X)$ (Lemma 2.3 (2)). Then the same is true for $\alpha_{i} \circ p$. The family of projections $\left\{\alpha_{i}\right\}_{i \in I}$ separates points of $X$. Now directly from Lemma 2.3(3) we conclude that $p \in \mathcal{F}(X, X)$.

If $X$ is a tame $G$-space, then $E(X)$ is also tame as a $G$-space. For every $G$-space $X$ there exists a maximal tame $G$-compactification (universal tame $G$-factor if $X$ is compact).

Definition 5.5. We say that a continuous function $f: X \rightarrow \mathbb{R}$ on a $G$-space $X$ is tame (notation $f \in$ Tame $(X)$ ) if it comes from a tame $G$-system.

Since the class of tame systems is closed under products and subsystems the collection $\operatorname{Tame}(X)$ is a $G$-subalgebra of $R U C(X)$ for every $G$-space $X$ (use, for example, the general approach as in [13, Prop. 2.9]). 
Proposition 5.6. Let $X$ be a compact $G$-space, $f \in C(X)$ and let $E^{f}=\operatorname{cls}_{p}(f G)$ be the pointwise closure of the orbit of $f$ in $\mathbb{R}^{X}$. The following conditions are equivalent:

(1) The function $f: X \rightarrow \mathbb{R}$ is tame.

(2) The cyclic $G$-space $X_{f}$ is tame.

(3) $c l s p(f G) \subset \mathcal{F}(X)$ (i.e. the orbit $f G$ is a Rosenthal family for $X$ ).

(4) For every countable subset $S \subset G, f S$ is a Rosenthal family for $X$.

Proof. The implication $(2) \Rightarrow(1)$ is obvious because $f$ comes from the cyclic $G$ space $X_{f}$.

$(1) \Rightarrow(3)$ : There exist: a tame compact $G$-system $X_{0}$, a $G$-quotient map $q$ : $X \rightarrow X_{0}$ and a function $f_{0} \in C\left(X_{0}\right)$ such that $f=f_{0} \circ q$. By Lemma 4.5(3) it suffices to show that $f_{0} G$ is a Rosenthal family for $X_{0}$. Clearly $f_{0} G$ is norm bounded in $C\left(X_{0}\right)$. We have to show that the corresponding pointwise closure of $f_{0} G=\left\{f_{0} \circ g: g \in G\right\} \subset \mathbb{R}^{X_{0}}$ is a subset of $\mathcal{F}\left(X_{0}\right)$. Observe that $\operatorname{cls}_{p}\left(f_{0} G\right)=$ $E^{f_{0}}:=\left\{f_{0} \circ p\right\}_{p \in E\left(X_{0}\right)}$. Our $G$-system $X_{0}$ is tame means that every $p: X_{0} \rightarrow X_{0}$ $\left(p \in E\left(X_{0}\right)\right)$ is fragmented. Thus every $f_{0} \circ p$ is also fragmented (because $f_{0}$ is uniformly continuous). So, $f_{0} G$ is a Rosenthal family for $X_{0}$.

$(3) \Rightarrow(2)$ : If $f G$ is a Rosenthal family for $X$, then $E^{f}=\operatorname{cls}_{p}(f G) \subset \mathcal{F}(X)$. This means that $f \circ p \in \mathcal{F}(X)$ for every $p \in E$. Consider the cyclic $G$-system $X_{f}$ and the natural $G$-quotient $\alpha_{f}: X \rightarrow X_{f}$. By elementary properties of cyclic $G$-spaces (Section 3) there exists a continuous function $f_{0}: X_{f} \rightarrow \mathbb{R}$ such that $f=f_{0} \circ \alpha_{f}$ and $f G=\left(f_{0} G\right) \circ \alpha_{f}$. By Lemma 4.5 we obtain that $f_{0} G$ is a Rosenthal family for $X_{f}$. Therefore, $f_{0} \circ p \in \mathcal{F}\left(X_{f}\right)$ for every $p \in E\left(X_{f}\right)$. Then also $f_{0} \circ g p=f_{0} g \circ p \in \mathcal{F}\left(X_{f}\right)$ for every $p \in E\left(X_{f}\right)$ and $g \in G$. Now since $f_{0} G$ separates points of $X_{f}$ (Section 3.1), by Lemma 2.3(3) we conclude that $p: X_{f} \rightarrow X_{f}$ is a fragmented map for every $p \in E\left(X_{f}\right)$. This means that $\left(G, X_{f}\right)$ is tame.

$(3) \Leftrightarrow(4)$ : Follows from Fact 4.3 .

Remark 5.7. By Rosenthal's dichotomy every bounded sequence in a Banach space either has a weak Cauchy subsequence or a subsequence equivalent to the unit vector basis of $l_{1}$ (the so-called $l_{1}$-sequence). Recall the definition of regularity of dynamical systems originally introduced by Köhler [26] for cascades in terms of independent sequences. A compact $G$-space $X$ is regular iff for every $f \in C(X)$ the orbit $f G$ does not contain an $l_{1}$-sequence (in other words, the second alternative is ruled out in Rosenthal's dichotomy). By Fact 4.3 it is equivalent to requiring that $f G$ be a Rosenthal family for $X$ for every $f \in C(X)$. In fact the notions of regularity and tameness coincide (see [10] (for metrizable systems) and Corollary 5.8 below).

Corollary 5.8. Let $X$ be a compact (not necessarily metrizable) G-space. The following conditions are equivalent:

(1) $(G, X)$ is a tame dynamical system (that is, $E(X) \subset \mathcal{F}(X, X)$ ).

(2) $C(X)=$ Tame $(X)$.

(3) $(G, X)$ is regular (in the sense of Köhler).

Proof. (2) $\Leftrightarrow(3)$ : Use Proposition 5.6 and Fact 4.3

(1) $\Leftrightarrow(2)$ : Observe that by standard arguments (see for example 13, Prop. 2.9]) the universal tame $G$-factor of $X$ is naturally isomorphic to $X$ iff $C(X)=$ Tame $(X)$. 
Let $X$ be a compact $G$-space. Then WAP functions on $X$ come from reflexively representable factors. Similarly, Asplund functions on a compact $G$-system $X$ are exactly functions which come from Asplund representable (that is, RN) factors. Every RN (being HNS) is tame in virtue of Lemma 5.3. Hence

$$
W A P(X) \subset A s p(X) \subset \operatorname{Tame}(X) .
$$

Another way to see these inclusions for metrizable $X$ is the following proposition (see also Lemma 4.4 and Section 1.3).

Proposition 5.9. Let $X$ be a compact metric $G$-space and $f \in C(X)$. Then

(1) $c l s p(f G) \subset C(X)$ if and only if $f \in W A P(X)$.

(2) cls $_{p}(f G)$ is a (compact) metrizable subspace in $\mathbb{R}^{X}$ iff $f \in A \operatorname{sp}(X)$ iff $f G$ is a fragmented family of functions on $X$.

(3) $\operatorname{cls}_{p}(f G) \subset \mathcal{B}_{1}(X)=\mathcal{F}(X)$ if and only if $f \in \operatorname{Tame}(X)$.

Proof. (1) Use Grothendieck's theorem: for a compact space $X$, a bounded subset $A \subset C(X)$ is relatively weakly compact in $C(X)$ iff it is pointwise relatively compact.

(2) By [13, we know that $f \in A s p(X)$ iff $f G$ is a fragmented family of functions on $X$. At the same time Lemma 4.4 shows that $\operatorname{cls}_{p}(f G)$ is a (compact) metrizable subspace in $\mathbb{R}^{X}$ iff $f G$ is a fragmented family of functions on $X$.

(3) Apply Proposition 5.6.

Remarks 5.10. (1) Note that the equivalence " $f \in A s p(X) \Leftrightarrow \operatorname{cls}_{p}(f G)$ is metrizable in $\mathbb{R}^{X}$ " is a new characterization of Asplund functions on metric compact $G$-systems.

(2) For a concrete example of a metric tame system which is not RN, see [13, Example 14.10]. 25].

Further results concerning tame systems can be found in [26, [10, [11, 20],

\section{THE MAIN RESUlts}

6.1. Banach representations of tame systems. Let us say that a compact $G$-space $X$ is Rosenthal representable if it admits a faithful representation on a Rosenthal Banach space (see Definition 4.7 above). Our main result (Theorem 6.9) asserts that a compact metric $G$-space is tame iff it is Rosenthal representable.

Theorem 6.1. Every Rosenthal representable (not necessarily metrizable) compact $G$-space is tame. In particular, the dynamical system $\left(I s o(V), B^{*}\right)$, where $B^{*}$ is the weak* compact unit ball of $V^{*}$, is tame for every Rosenthal Banach space $V$.

Proof. It is enough to show that for every Rosenthal Banach space $V$ the associated dynamical system $\left(I s o(V), B^{*}\right)$ is tame. We have to show that $p: B^{*} \rightarrow B^{*}$ is a fragmented map for every $p \in E\left(G, B^{*}\right)$.

Claim. For every $p \in E\left(G, B^{*}\right)$ there exists a (uniquely defined) linear operator $\bar{p}: V^{*} \rightarrow V^{*}$ with the norm $\leq 1$ such that $p=\left.\bar{p}\right|_{B^{*}}$.

Proof. Consider the enveloping semigroup $E\left(G, V^{*}\right)$ of the separately continuous action $G \times V^{*} \rightarrow V^{*}$, where $V^{*}$ carries the weak* topology. That is, as in the case of compact $G$-spaces (Section 3.2) $E\left(G, V^{*}\right)$ is the pointwise closure $E\left(G, V^{*}\right)$ of the 
set of all $g$-translations $\breve{G}=\left\{\breve{g}: V^{*} \rightarrow V^{*}\right\}_{g \in G}$. Then $E\left(G, V^{*}\right)$ is a compact right topological semigroup. The compactness follows from the fact that the $G$-orbits in $V^{*}$ are relatively weak* compact. Furthermore, every $t \in E\left(G, V^{*}\right)$ (as a pointwise limit of linear isometries) is a linear operator $V^{*} \rightarrow V^{*}$ with norm $\leq 1$. Consider the natural continuous restriction $\Psi: E\left(G, V^{*}\right) \rightarrow E\left(G, B^{*}\right),\left.t \mapsto t\right|_{B^{*}}$. Since $E\left(G, V^{*}\right)$ is compact it easily follows that $\Psi$ is onto. In fact $\Psi$ is even injective (hence, a homeomorphism) because every linear operator $V^{*} \rightarrow V^{*}$ is defined uniquely by its restriction to $B^{*}$.

Let $p \in E\left(G, B^{*}\right)$. According to the claim, $p=\left.\bar{p}\right|_{B^{*}}$, where $\bar{p}$ is a linear operator $\bar{p}: V^{*} \rightarrow V^{*}$ with norm $\leq 1$. Then, for every vector $f \in V$, the composition $f \circ \bar{p}: V^{*} \rightarrow \mathbb{R}$ is a linear bounded (hence norm continuous) functional on $V^{*}$. That is, $f \circ \bar{p} \in V^{* *}$ belongs to the second dual. By the reformulation of a theorem of E. Saab and P. Saab (see Fact 4.11 and Remark 4.13(1)) mentioned above, the corresponding restriction $\left.(f \circ \bar{p})\right|_{B^{*}}: B^{*} \rightarrow \mathbb{R}$ is a fragmented function. Next note that $V$ separates points of $B^{*}$. Since $f \circ p=\left.(f \circ \bar{p})\right|_{B^{*}}$ is fragmented for every $f \in V$, we can apply Lemma 2.3(3). It follows that $p: B^{*} \rightarrow B^{*}$ is fragmented, completing the proof of the theorem.

Lemma 6.2. Let $X$ be a compact metric $G$-space. The following conditions are equivalent:

(1) $(G, X)$ is representable on a separable Rosenthal Banach space.

(2) $(G, X)$ admits countably many representations on separable Rosenthal Banach spaces which separate points of $X$.

Proof. Observe that the $l_{2}$-sum of a sequence of separable Rosenthal Banach spaces is again a separable Rosenthal space. Indeed, this follows for instance from the equivalence of (1) and (2) in Odell and Rosenthal's Theorem (Fact 4.10). The rest is similar to the proof of [30, Lemma 4.9].

Theorem 6.3. Let $X$ be a compact $G$-space, $F \subset C(X)$ a Rosenthal family for $X$ such that $F$ is $G$-invariant (that is, $f G \subset F \quad \forall f \in F$ ). Then:

(1) There exist: a Rosenthal Banach space $V$, an injective mapping $\nu: F \rightarrow B_{V}$ into the unit ball $B_{V}$ and a continuous representation

$$
h: G \rightarrow I s o(V), \quad \alpha: X \rightarrow V^{*}
$$

of $(G, X)$ on $V$ such that $\alpha$ is a weak* continuous map (topological embedding if $F$ separates points of $X)$ and

$$
f(x)=\langle\nu(f), \alpha(x)\rangle \quad \forall f \in F \quad \forall x \in X .
$$

Thus the following diagram commutes:

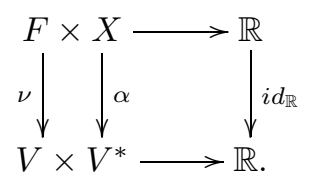


(2) If $X$ is metrizable, then in addition we can suppose that $V$ is separable and there exists a homeomorphic embedding

$$
\nu_{0}: K:=\operatorname{cls} p(F) \hookrightarrow V^{* *}
$$

furnishing $V^{* *}$ with its weak $k^{*}$ topology and the following diagram commutes:

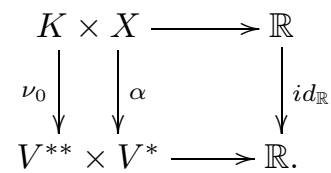

Proof. (1) Let $W$ be the symmetrized convex hull of $F$; that is,

$$
W:=\operatorname{co}(F \cup-F) \text {. }
$$

Claim 1. $W$ is also a Rosenthal family for $X$.

Proof. It is easy to see that $F \cup-F$ is a Rosenthal family for $X$. Now apply Proposition 4.15

For brevity of notation let $\mathcal{A}:=C(X)$ denote the Banach space $C(X), B$ will denote its unit ball, and $B^{*}$ will denote the weak* compact unit ball of the dual space $\mathcal{A}^{*}=C(X)^{*}$.

Claim 2. $W$ is a Rosenthal family for $B^{*}$.

Proof. Apply Proposition 4.19.

Consider the sequence of sets $M_{n}:=2^{n} W+2^{-n} B$. Since $W$ is convex and symmetric, we can apply the construction of Davis-Figiel-Johnson-Pelczyński [4] as follows. Let $\|\cdot\|_{n}$ be the Minkowski functional of the set $M_{n}$. That is,

$$
\|v\|_{n}=\inf \left\{\lambda>0 \mid v \in \lambda M_{n}\right\} .
$$

Then $\|\cdot\|_{n}$ is a norm on $\mathcal{A}$ equivalent to the given norm of $\mathcal{A}$. For $v \in \mathcal{A}$, set

$$
N(v):=\left(\sum_{n=1}^{\infty}\|v\|_{n}^{2}\right)^{1 / 2} \text { and } V:=\{v \in \mathcal{A} \mid N(v)<\infty\} .
$$

Denote by $j: V \hookrightarrow \mathcal{A}$ the inclusion map. Then $(V, N)$ is a Banach space, $j: V \rightarrow \mathcal{A}$ is a continuous linear injection and

$$
W \subset j\left(B_{V}\right)=B_{V} .
$$

Indeed, if $v \in W$, then $2^{n} v \in M_{n}$; hence $\|v\|_{n} \leq 2^{-n}$ and $N(v)^{2} \leq \sum_{n \in \mathbb{N}} 2^{-2 n}<1$.

The given action $G \times X \rightarrow X$ induces the natural linear norm-preserving continuous right action $C(X) \times G \rightarrow C(X)$ on the Banach space $\mathcal{A}=C(X)$. It follows by the construction that $W$ and $B$ are $G$-invariant subsets in $\mathcal{A}$. This implies that $V$ is a $G$-invariant subset of $\mathcal{A}$ and the restricted natural linear action $V \times G \rightarrow V, \quad(v, g) \mapsto v g$ is norm-preserving, that is, $N(v g)=N(v)$. Moreover, by the definition of the norm $N$, we can show that this action is norm continuous (use the fact that, for each $n \in \mathbb{N}$, the norm $\|\cdot\|_{n}$ on $\mathcal{A}$ is equivalent to the given norm on $\mathcal{A})$. Therefore, the co-homomorphism $h: G \rightarrow \operatorname{Iso}(V), h(g)(v):=v g$ is well defined and continuous.

Let $j^{*}: \mathcal{A}^{*} \rightarrow V^{*}$ be the adjoint map of $j: V \rightarrow \mathcal{A}$. Define $\alpha: X \rightarrow V^{*}$ as follows. For every $x \in X \subset C(X)^{*}$ set $\alpha(x)=j^{*}(x)$. Then $(h, \alpha)$ is a continuous representation of $(G, X)$ on the Banach space $V$. 

Then

By the construction $F \subset W \subset B_{V}$. Define $\nu: F \hookrightarrow B_{V}$ as the natural inclusion.

$$
f(x)=\langle\nu(f), \alpha(x)\rangle \quad \forall f \in F \quad \forall x \in X .
$$

It follows in particular that if $F$ separates points of $X$, then $\alpha$ is an embedding.

Claim 3. $j\left(B_{V}\right) \subset \bigcap_{n \in \mathbb{N}} M_{n}=\bigcap_{n \in \mathbb{N}}\left(2^{n} W+2^{-n} B\right)$.

Proof. The norms $\|\cdot\|_{n}$ on $\mathcal{A}$ are equivalent to each other. It follows that if $v \in B_{V}$, then $\|v\|_{n}<1$ for all $n \in \mathbb{N}$. That is, for every $n \in \mathbb{N}, v \in \lambda_{n} M_{n}$ for some $0<\lambda_{n}<1$. By the construction, $M_{n}$ is a convex subset containing the origin. This implies that $\lambda_{n} M_{n} \subset M_{n}$. Hence $j(v)=v \in M_{n}$ for every $n \in \mathbb{N}$.

Claim 4. The set $\bigcap_{n \in \mathbb{N}} M_{n}$ (and hence also its subset $j\left(B_{V}\right)$ ) is sequentially precompact in the second dual $\left(\mathcal{A}^{* *}, \sigma\left(\mathcal{A}^{* *}, \mathcal{A}^{*}\right)\right)$ (i.e. $\mathcal{A}^{* *}$ endowed with its weak* topology).

Proof. We use the argument of [4, Lemma 1 (xii), p. 323] with some minor changes.

Let $\left\{c_{n}\right\}_{1}^{\infty}$ be a sequence in $\bigcap_{k \in \mathbb{N}} M_{k}$. Then for each fixed $n \in \mathbb{N}$ and every $k \in \mathbb{N}$ we can represent $c_{n}$ as

$$
c_{n}=2^{k} w_{n}^{k}+2^{-k} b_{n}^{k}
$$

with $w_{n}^{k} \in W, b_{n}^{k} \in B$. By Claim 2 we know that $W$ is a Rosenthal family for $B^{*}$. Thus by Fact $4.3, W$ is sequentially precompact in $\mathbb{R}^{B^{*}}$, hence also in $\mathbb{R}^{C(X)^{*}}$. It follows that $W$ is sequentially precompact in $\left(\mathcal{A}^{* *}, \sigma\left(\mathcal{A}^{* *}, \mathcal{A}^{*}\right)\right)($ as $\mathcal{A}=C(X))$. Applying a diagonal process we can choose a subsequence $\left\{n_{i}\right\}_{i=1}^{\infty}$ such that for each $k$ the sequence $\left\{w_{n_{i}}^{k}\right\}_{i=1}^{\infty}$ is $\sigma\left(\mathcal{A}^{* *}, \mathcal{A}^{*}\right)$-convergent to an element, say, $x_{k}^{* *} \in \mathcal{A}^{* *}$. In order to simplify our notation we will relabel our sequences and now assume that for every $k$,

$$
w^{*}-\lim _{n} w_{n}^{k}=x_{k}^{* *}
$$

in $\mathcal{A}^{* *}$.

Claim 5. The sequence $2^{k} x_{k}^{* *}$ is norm Cauchy in the second dual $\mathcal{A}^{* *}$.

Proof. Note first that by (6.2) we have

$$
\left\|2^{k} w_{n}^{k}-2^{l} w_{n}^{l}\right\|=\left\|2^{-k} b_{n}^{k}-2^{-l} b_{n}^{l}\right\| \leq 2 \cdot 2^{-\min \{k, l\}} .
$$

Now for a fixed unit vector $x^{*} \in \mathcal{A}^{*}$ we have

$$
\begin{aligned}
& \left|\left\langle x^{*}, 2^{k} x_{k}^{* *}-2^{l} x_{l}^{* *}\right\rangle\right| \\
& \quad \leq\left|\left\langle x^{*}, 2^{k} x_{k}^{* *}-2^{k} w_{n}^{k}\right\rangle\right|+\left|\left\langle x^{*}, 2^{k} w_{n}^{k}-2^{l} w_{n}^{l}\right\rangle\right\rangle|+|\left\langle x^{*}, 2^{l} w_{n}^{l}-2^{k} x_{l}^{* *}\right\rangle \mid \\
& \quad \leq\left|\left\langle x^{*}, 2^{k} x_{k}^{* *}-2^{k} w_{n}^{k}\right\rangle\right|+2 \cdot 2^{-\min \{k, l\}}+\left|\left\langle x^{*}, 2^{l} w_{n}^{l}-2^{k} x_{l}^{* *}\right\rangle\right| .
\end{aligned}
$$

By (6.3) and (6.4) we get

$$
\left|\left\langle x^{*}, 2^{k} x_{k}^{* *}-2^{l} x_{l}^{* *}\right\rangle\right| \leq 2 \cdot 2^{-\min \{k, l\}} ;
$$

hence

$$
\left\|2^{k} x_{k}^{* *}-2^{l} x_{l}^{* *}\right\|=\sup _{\left\|x^{*}\right\|=1}\left|\left\langle x^{*}, 2^{k} x_{k}^{* *}-2^{l} x_{l}^{* *}\right\rangle\right| \leq 2 \cdot 2^{-\min \{k, l\}} .
$$

We let $\xi:=\lim _{k \rightarrow \infty} 2^{k} x_{k}^{* *}$ in the Banach space $\mathcal{A}^{* *}$. 
Claim 6. $w^{*}-\lim _{n \rightarrow \infty} c_{n}=\xi$; i.e. $\left\{c_{n}\right\}_{n=1}^{\infty}$ is $\sigma\left(\mathcal{A}^{* *}, \mathcal{A}^{*}\right)$-convergent to $\xi \in \mathcal{A}^{* *}$.

Proof. Again fix a unit vector $x^{*} \in \mathcal{A}^{*}$. Given $\varepsilon>0$ fix $k_{0} \in \mathbb{N}$ such that $2^{-k_{0}}<\varepsilon$, and

$$
\left\|2^{k} x_{k}^{* *}-\xi\right\|<\varepsilon
$$

for $k>k_{0}$. By (6.2), $\left\|c_{n}-2^{k} w_{n}^{k}\right\| \leq 2^{-k}$; hence for $k>k_{0}$ and every $n \in \mathbb{N}$ we get

$$
\begin{aligned}
& \left|\left\langle x^{*}, c_{n}-\xi\right\rangle\right| \\
& \quad \leq\left|\left\langle x^{*}, c_{n}-2^{k} w_{n}^{k}\right\rangle\right|+\left|\left\langle x^{*}, 2^{k} w_{n}^{k}-2^{k} x_{k}^{* *}\right\rangle\right|+\left|\left\langle x^{*}, 2^{k} x_{k}^{* *}-\xi\right\rangle\right| \\
& \quad \leq \varepsilon+\left|\left\langle x^{*}, 2^{k} w_{n}^{k}-2^{k} x_{k}^{* *}\right\rangle\right|+\varepsilon .
\end{aligned}
$$

For an arbitrary but fixed $k>k_{0}$, by (6.3) again, there is an $n_{0} \in \mathbb{N}$ such that for $n>n_{0}$,

$$
\left|\left\langle x^{*}, 2^{k} w_{n}^{k}-2^{k} x_{k}^{* *}\right\rangle\right|<\varepsilon .
$$

Combining these inequalities we see that for $n>n_{0}$,

$$
\left|\left\langle x^{*}, c_{n}-\xi\right\rangle\right| \leq 3 \varepsilon
$$

Note that now we have completed the proof of Claim 4 .

Let $\overline{B_{V}}$ be the $\sigma\left(V^{* *}, V^{*}\right)$-closure of the unit ball $B_{V}$ in $V^{* *}$ (in fact $\overline{B_{V}}$ is the unit ball of the second dual $V^{* *}$ by Goldstine's theorem). Similarly, $\overline{j^{* *}\left(B_{V}\right)}=$ $\overline{j\left(B_{V}\right)}$ denotes the $\sigma\left(\mathcal{A}^{* *}, \mathcal{A}^{*}\right)$-closure in the second dual $\mathcal{A}^{* *}$. From Claim 4 we know that $j\left(B_{V}\right)$ is sequentially precompact in $\overline{j\left(B_{V}\right)}$. In order to show that $V$ is a Rosenthal space we have to establish that $B_{V}$ is a Rosenthal family for $B^{*}$ (Lemma 4.9). In turn, this is equivalent by Fact 4.3 to showing that $B_{V}$ is sequentially precompact in $\mathbb{R}^{B^{*}}$, or equivalently in the $\sigma\left(V^{* *}, V^{*}\right)$-compact space $\overline{B_{V}}$.

It is enough to find a homeomorphism $\eta: \overline{B_{V}} \rightarrow \overline{j\left(B_{V}\right)}$ such that $\eta(b)=j(b)$ for every $b \in B_{V}$. Consider the canonical second adjoint map

$$
j^{* *}: V^{* *} \rightarrow \mathcal{A}^{* *} \text {. }
$$

This map is injective by [4, Lemma 1(iii)]. Using the compactness of $j^{* *}\left(\overline{B_{V}}\right)$ we get $j^{* *}\left(\overline{B_{V}}\right)=\overline{j^{* *}\left(B_{V}\right)}=\overline{j\left(B_{V}\right)}$. Now the required homeomorphism $\eta$ is the restriction of $j^{* *}$ to the $\sigma\left(V^{* *}, V^{*}\right)$-compact space $\overline{B_{V}}$.

We obtain that $V$ is a Rosenthal space and the assertion (1) is proved.

(2) If the compact space $X$ is metrizable, then $C(X)$ is separable and it is also easy to see that $(V, N)$ is separable. The pointwise closure $K:=\operatorname{cls}_{p}(F)$ of $F$ in $\mathbb{R}^{X}$ is a subset of $b \mathcal{B}_{1}(X)$. Consider now the mapping $T: b \mathcal{B}_{1}(X) \rightarrow C(X)^{* *}$ (see Remark 4.16). This map induces by Proposition 4.18 a homeomorphic embedding

$$
T: K=\operatorname{cls}_{p}(F) \rightarrow \operatorname{cls}_{p}(T(F)) \subset b \mathcal{B}_{1}\left(B^{*}\right) \cap C(X)^{* *}
$$

of $K$ into $\mathcal{A}^{* *}=C(X)^{* *}$.

By construction $F$ is a subset of $V$. Consider the second adjoint map

$$
j^{* *}: V^{* *} \rightarrow \mathcal{A}^{* *} .
$$

Recall again that this map is injective by [4, Lemma 1 (iii)]. Therefore $j^{* *}$ induces a homeomorphism between the weak* compact spaces $\operatorname{cls}_{V^{* *}}(F)$ and $\operatorname{cls}_{\mathcal{A}^{* *}}(F)$. Summing up we can define the desired homeomorphic embedding as follows:

$$
\nu_{0}: K \rightarrow V^{* *}, \quad f \mapsto\left(j^{* *}\right)^{-1}(T(f)) .
$$


Observe that $\nu_{0}(f)=\nu(f)$ for every $f \in F$. Since $F=\nu(F)$ is pointwise dense in $K$, using (6.1), we get

$$
f(x)=\left\langle\nu_{0}(f), \alpha(x)\right\rangle \quad \forall f \in K, \quad \forall x \in X .
$$

So, Theorem 6.3 is proved.

Recall again (see Section 3.3.2) that a compact space $X$ is called Radon-Nikodým $(\mathrm{RN})$ if $X$ is homeomorphic to a weak* compact subset of the dual $V^{*}$ for an Asplund space $V$. A well-known result characterizes Rosenthal spaces as those Banach spaces whose dual has the weak Radon-Nikodým property 42, Corollary 7.3.8]. It is therefore natural to introduce the following definition.

Definition 6.4. We say that a compact topological space $X$ is weakly RadonNikodym (WRN) if $X$ is homeomorphic to a weak* compact subset of the dual $V^{*}$ of a Rosenthal space $V$. This definition agrees with the definition of WRN subsets (cf. Remark 4.13(2)). A compact $G$-space $X$ is called a WRN $G$-space if $X$, as a $G$-space, is Rosenthal representable.

As a corollary of Theorem 6.3 we get the following characterization of WRN compacta.

Theorem 6.5. Let $X$ be a compact space. The following conditions are equivalent:

(1) $X$ is WRN.

(2) There exists a Rosenthal family $F \subset C(X)$ of $X$ which separates the points of $X$.

Remark 6.6. We mention without proof that a compact $G$-space $X$ is RN (that is, Asplund representable) if and only if there exists a bounded subset $F \subset C(X)$ such that $F$ separates points of $X, F$ is $G$-invariant and $F$ is a fragmented family of functions (Definition 2.7). Comparing this with Theorem 6.10 below we see that there exists a complete analogy between RN-systems and fragmented families on one side and WRN-systems and Rosenthal families on the other.

Next we present a characterization of tame functions in terms of Banach representations.

Theorem 6.7. Let $X$ be a compact $G$-space. The following conditions are equivalent:

(1) $f: X \rightarrow \mathbb{R}$ is tame.

(2) $f: X \rightarrow \mathbb{R}$ comes from a Rosenthal Banach space. That is, there exist a continuous representation $(h, \alpha)$ of $(G, X)$ on a Rosenthal Banach space $V$ and a vector $v \in V$ such that

$$
f(x)=\langle v, \alpha(x)\rangle \quad \forall x \in X .
$$

If $X$ is metrizable we can suppose in addition in (2) that $V$ is separable.

Proof. $(2) \Rightarrow(1)$ : Directly follows by Theorem 6.1.

$(1) \Rightarrow(2)$ : Let $f \in$ Tame $(X)$. This means By Proposition 5.6 that the orbit $f G$ is a Rosenthal family for $X$. Now we can apply Theorem 6.3 to the family $F:=f G$. 
Here are the promised Banach space characterizations of tame and metrizable tame systems.

Theorem 6.8. Let $X$ be a compact $G$-space. The following conditions are equivalent:

(1) $(G, X)$ is a tame $G$-system.

(2) $(G, X)$ is Rosenthal approximable.

Proof. $(2) \Rightarrow(1)$ : Apply again Theorem 6.1 and take into account that the class of tame $G$-systems is closed under subsystems and arbitrary products for every given $G$ (Lemma 5.4).

$(1) \Rightarrow(2)$ : First of all note that $C(X)=$ Tame $(X)$ by Corollary 5.8 Applying Theorem 6.7 we conclude that every $f \in C(X)=$ Tame $(X)$ on a compact $G$-space $X$ comes from a Rosenthal representation. Continuous functions separate points of $X$. This implies that there exist sufficiently many Rosenthal representations of $(G, X)$.

Theorem 6.9. Let $X$ be a compact metric $G$-space. The following conditions are equivalent:

(1) $(G, X)$ is tame.

(2) $(G, X)$ is representable on a separable Rosenthal Banach space.

Proof. (2) $\Rightarrow(1)$ : Apply once again Theorem 6.1

$(1) \Rightarrow(2)$ : Since the compact space $X$ is metrizable there is a sequence of functions $f_{n} \in C(X)=\operatorname{Tame}(X)$ which separates points of $X$. For each $f_{n}$ we can construct by Theorem 6.7 a continuous Rosenthal representation $\left(h_{n}, \alpha_{n}\right)$ of $(G, X)$ such that our original function $f_{n}$ comes from the system $\left(G, \alpha_{n}(X)\right)$. Applying Lemma 6.2 we conclude that $(G, X)$ is Rosenthal representable.

If $X$ is a tame, not necessarily metrizable, dynamical $G$-system, then the induced systems $\left(G, B^{*}\right)$ (on the weak* compact unit ball $B^{*}$ of $\left.C(X)^{*}\right)$ and $(G, P(X))$ (where $P(X)$ denotes the weak* compact subspace of $B^{*}$ consisting of all probability measures on $X$ ) are tame as well (see Corollary 6.11). For metrizable $X$ this is [10, Theorem 1.5]. In fact one may show a stronger result:

Theorem 6.10. Let $X$ be a compact $G$-space. The following conditions are equivalent:

(1) $(G, X)$ is Rosenthal representable (i.e. $X$ is a WRN G-space).

(2) There exists a $G$-invariant Rosenthal family $A \subset C(X)$ for $X$ which separates points of $X$.

(3) $\left(G, B^{*}\right)$ is Rosenthal representable.

(4) $(G, P(X))$ is Rosenthal representable.

Proof. (1) $\Leftrightarrow(2)$ : Apply Theorem 6.3 and Lemma 4.9.

$(2) \Rightarrow(3)$ : Let $A$ be a $G$-invariant point separating the Rosenthal family for $X$. We will show that there exists a $G$-invariant point separating the Rosenthal family for $B^{*}$. Produce inductively the sequence $A_{n}:=A_{1} \cdot A_{1} \cdots A_{1}$ ( $n$-times), where $A_{1}:=A$. We can suppose that $A \subset B_{C(X)}$ and contains the constant function 1. We can show by diagonal arguments (use Fact 4.3 or Proposition 4.6) that $A_{n}$ is also a Rosenthal family for $X$. Furthermore (by the same results) it is easy to show 
that the $G$-invariant family $M:=\bigcup_{n} 2^{-n} A_{n}$ is Rosenthal for $X$. By the StoneWeierstrass Theorem the algebra $\operatorname{span}(M)$ (linear span of $M$ in $C(X)$ ) is dense in $C(X)$. This implies that $M$ itself separates the points of $B^{*}$. By Proposition 4.19, $M$ is a ( $G$-invariant) Rosenthal family for $B^{*}$. Now we apply the part $(1) \Leftrightarrow(2)$ to the case of $\left(G, B^{*}\right)$.

$(3) \Rightarrow(4)$ and $(4) \Rightarrow(1)$ are obvious.

Theorem 6.11. Let $X$ be a compact $G$-space. Then $(G, X)$ is tame iff $\left(G, B^{*}\right)$ (equivalently, $(G, P(X)))$ is tame.

Proof. It is enough to show that $\left(G, B^{*}\right)$ is tame for every tame system $(G, X)$. By Theorem 6.10 we know that $X$ is $G$-embedded into a $G$-product $\prod_{i \in I} X_{i}$ of Rosenthal representable $G$-systems $X_{i}$. The corresponding $G$-system $B_{i}^{*}$ is Rosenthal representable, hence tame by virtue of Theorems 6.8 and 6.1. Now observe that $B^{*}$ is naturally $G$-embedded into the $G$-product $\prod_{i \in I} B_{i}^{*}$ and use Lemma 5.4 .

Theorem 6.12. Let $G$ be a topological group. The following conditions are equivalent:

(1) $f: G \rightarrow \mathbb{R}$ is tame.

(2) $f$ is a matrix coefficient of a continuous co-representation of $G$ on a Rosenthal space. That is, there exist: a Rosenthal space $V$, a continuous cohomomorphism $h: G \rightarrow I s o(V)$, vectors $v \in V$ and $\psi \in V^{*}$ such that $f(g)=\psi(v g)$ for every $g \in G$.

Proof. (1) $\Rightarrow(2)$ : Choose a tame $G$-compactification $\nu: G \rightarrow X$ of $G$ and a continuous function $f_{0}: X \rightarrow \mathbb{R}$ such that $f=f_{0} \circ \nu$. Now we can apply Theorem 6.7 to $f_{0}$ getting the desired $V$ and vectors $v$ and $\psi:=\alpha(\nu(e))$.

$(2) \Rightarrow(1)$ : Apply Theorem 6.1

6.2. Compact spaces in the second dual of Rosenthal spaces. Again we remind the reader that a compact topological space $K$ is Rosenthal if it is homeomorphic to a pointwise compact subset of the space $\mathcal{B}_{1}(X)$ of functions of the first Baire class on a Polish space $X$.

Definition 6.13. (1) We say that a compact space $K$ is strongly Rosenthal if $K$ is a subspace of $\mathcal{B}_{1}(X)$ with compact metrizable $X$.

(2) We say that a compact space $K$ is an admissible Rosenthal compactum (or simply admissible) if there exists a compact metric space $X$ and a Rosenthal family $F$ for $X$ such that $K \subset \operatorname{cls}_{p}(F)$.

In the second definition it follows that $K \subset \operatorname{cls}_{p}(F) \subset \mathcal{B}_{1}(X)$. Hence every admissible compactum is a strongly Rosenthal compactum. Clearly every strongly Rosenthal compact space is Rosenthal.

Every subset $F \subset C(X)$ is norm separable for a compact metric $X$. Hence such an $F$ is also separable with respect to the pointwise convergence topology. Thus in Definition 6.13 (2), we can assume that $F$ is countable.

Pol's example, mentioned in the introduction, shows that not every separable Rosenthal compactum is strongly Rosenthal (and a fortiori also not admissible).

Lemma 6.14. The classes of Rosenthal, strongly Rosenthal and admissible compact spaces are closed under the operations of passing to closed subspaces and taking countable products. 
Proof. The hereditary property of each of these classes is obvious. In order to see that the countable product $K:=\prod_{n} K_{n}$ of Rosenthal compact spaces $K_{n}$ is again Rosenthal we consider the topological (disjoint) sum $X:=\coprod_{n \in \mathbb{N}} X_{n}$, where $X_{n}$ is a Polish space for which $K_{n} \subset \mathcal{B}_{1}\left(X_{n}\right)$. Then $K$ can be embedded into $\mathcal{B}_{1}(X)$ as follows. For each element

$$
f:=\left(f_{1}, f_{2}, \cdots\right) \in \prod K_{n}=K,
$$

there exists a uniquely defined function $j(f): X \rightarrow \mathbb{R}$ such that the restriction of $j(f)$ on $X_{n}$ is exactly $f_{n}$. Clearly, $j(f)$ is a Baire 1 function on $X$. This defines the continuous map $j: K \rightarrow \mathcal{B}_{1}(X)$. Since $j$ is injective and $K$ is compact we conclude that $j$ is a topological embedding.

Suppose now that each $K_{n}$ is strongly Rosenthal. Then, by definition, we can assume in addition that each $X_{n}$ as above is a compact metric space. Now it is easy to see that $K$ admits a topological embedding into $\mathcal{B}_{1}\left(X^{*}\right)$, where $X^{*}:=X \cup\{\infty\}$ is the one-point compactification of $X=\coprod_{n \in \mathbb{N}} X_{n}$. In this case we define $j^{*}: K \rightarrow$ $\mathcal{B}_{1}\left(X^{*}\right)$ by $j^{*}(f)(\infty)=0$ and $j^{*}(f)(x)=j(f)(x)$ for every $x \in X$. Then again $j^{*}$ is well defined and it embeds $K$ into $\mathcal{B}_{1}\left(X^{*}\right)$.

Finally we consider the case where each $K_{n}$ is admissible. As in the second case we have the topological embedding

$$
j^{*}: K \hookrightarrow \mathcal{B}_{1}\left(X^{*}\right) .
$$

We have to show that there exists a family $F \subset C\left(X^{*}\right)$ such that $j^{*}(K) \subset \operatorname{cls}_{p}(F)$. For each $n \in \mathbb{N}$ fix a countable subset $F_{n} \subset C\left(X_{n}\right)$ such that $K_{n} \subset \operatorname{cls}_{p}\left(F_{n}\right)$. It is enough to show our assertion in the case where $\operatorname{cls}_{p}\left(F_{n}\right)=K_{n}$. For each $k \in \mathbb{N}$ consider the elements of the type

$$
f:=\left(f_{1}, f_{2}, \cdots, f_{k}, 0_{k+1}, 0_{k+2}, \cdots\right) \in \prod K_{n}=K,
$$

where $f_{i} \in F_{i}$ for every $i \leq k$ and each $0_{k+m}$ denotes the constant zero function on $X_{k+m}$ (again without restriction of generality we can assume that $0_{t} \in F_{t}$ for every $t \in \mathbb{N}$ ). Varying $k \in \mathbb{N}$ and $f_{i} \in F_{i}$ with $i \leq k$ we get a countable subset $F_{0} \subset K$. Clearly this subset is dense in the product space $K=\prod_{n \in \mathbb{N}} K_{n}$. It is easy to see that its image $F:=j^{*}\left(F_{0}\right)$ is the required family. That is, $F \subset C\left(X^{*}\right)$ and $j^{*}(K) \subset \operatorname{cls}_{p}(F)$.

Proposition 6.15. Let $X$ be a compact metric $G$-space. The following conditions are equivalent:

(1) $(G, X)$ is a tame system.

(2) $E(X)$ is an admissible Rosenthal compactum.

(3) $E(X)$ is a Rosenthal compactum.

Proof. (1) $\Rightarrow(2)$ : Let $(G, X)$ be a tame system. Then every continuous function $f \in C(X)$ is tame. This means that $f G$ is a Rosenthal family for $X$. Then the compact space $E^{f}:=\operatorname{cls}_{p}(f G)$ is a subset of $\mathcal{B}_{1}(X)$ (Proposition 5.6 and Corollary 2.6(2)). So the compactum $E^{f}$ is admissible. Since $X$ is a metrizable compact space one may choose a countable set of functions $\left\{f_{m}\right\}_{m \in \mathbb{N}}$ which separates the points in $X$. Then $E(X)$ can be naturally embedded into the countable product $K:=\prod_{m} E^{f_{m}}$, which is admissible by Lemma 6.14

$(2) \Rightarrow(3)$ : is clear.

$(1) \Leftrightarrow(3)$ : Follows directly from the definitions. 
We have the following related purely topological result:

Theorem 6.16. Let $K$ be a compact space. The following conditions are equivalent:

(1) $K$ is an admissible Rosenthal compactum.

(2) $K$ is homeomorphic to a weak $k^{*}$ closed subset in the second dual of a separable Rosenthal Banach space $V$.

Proof. $(1) \Rightarrow(2)$ : Let $K$ be an admissible compactum. By Definition 6.13 there exists a compact metric space $X$ and a Rosenthal family $F \subset C(X)$ such that $K \subset \operatorname{cls}_{p}(F) \subset \mathcal{B}_{1}(X)$. We have to show that $K$ is homeomorphic to a weak* closed subset in the second dual of a separable Rosenthal Banach space $V$. It is enough to establish this for the case of $K=\operatorname{cls}_{p}(F)$. But this fact follows directly from Theorem 6.3(2) when one considers a trivial (identity) $G$-action on $X$.

$(2) \Rightarrow(1)$ : We have to show that $K$ is an admissible compactum. It is enough to show this for the particular case where $K:=B^{* *}:=B_{V^{* *}}$, the unit ball in the second dual. Since $V$ is separable, $X:=B^{*}$, the weak* compact unit ball in $V^{*}$, is a metrizable compact space. By our assumption $V$ is a Rosenthal space. Then by Fact 4.10 (3), $K=B^{* *}$ is naturally embedded into $\mathcal{B}_{1}(X)$ with $X:=B^{*}$. By Goldstine's theorem the unit ball $B:=B_{V}$ of $V$ is weak ${ }^{*}$-dense in $B^{* *}$. At the same time $B$ can be treated as a (bounded) subset of $C(X)$. Thus, $B$ is a Rosenthal family for $X$. Hence the compactum $K$ is admissible in the sense of Definition 6.13(2).

\section{REFERENCES}

1. E. Akin, J. Auslander, and K. Berg, Almost equicontinuity and the enveloping semigroup, Topological dynamics and applications, Contemporary Mathematics 215, a volume in honor of R. Ellis, 1998, pp. 75-81. MR1603149 (99k:54032)

2. J. Bourgain, D.H. Fremlin and M. Talagrand, Pointwise compact sets in Baire-measurable functions, Amer. J. of Math., 100:4 (1977), 845-886. MR509077 (80b:54017)

3. R.D. Bourgin, Geometric Aspects of Convex Sets with the Radon-Nikodým Property, Lecture Notes in Math. 993, Springer-Verlag, 1983. MR704815 (85d:46023)

4. W.J. Davis, T. Figiel, W.B. Johnson and A. Pelczyński, Factoring weakly compact operators, J. of Funct. Anal., 17 (1974), 311-327. MR0355536 (50:8010)

5. D. van Dulst, Characterization of Banach spaces not containing $l^{1}$, Centrum voor Wiskunde en Informatica, Amsterdam, 1989. MR1002733 (90h:46037)

6. R. Ellis, Lectures on Topological Dynamics, W. A. Benjamin, Inc., New York, 1969. MR.0267561 (42:2463)

7. R. Ellis and M. Nerurkar, Weakly almost periodic flows, Trans. Amer. Math. Soc. 313, (1989), 103-119. MR930084 (89i:28010)

8. R. Engelking, General topology, Heldermann Verlag, Berlin, 1989. MR.1039321 (91c:54001)

9. M. Fabian, Gateaux differentiability of convex functions and topology. Weak Asplund spaces, Canadian Math. Soc. Series of Monographs and Advanced Texts, A Wiley-Interscience Publication, New York, 1997. MR1461271 (98h:46009)

10. E. Glasner, On tame dynamical systems, Colloq. Math. 105 (2006), 283-295. MR 2237913 (2007d:37005)

11. E. Glasner, The structure of tame minimal dynamical systems, Ergod. Th. and Dynam. Sys. 27, (2007), 1819-1837. MR2371597 (2008m:37015)

12. E. Glasner, Enveloping semigroups in topological dynamics, Topology Appl. 154, (2007), 2344-2363. MR2328017 (2008f:37021)

13. E. Glasner and M. Megrelishvili, Hereditarily non-sensitive dynamical systems and linear representations, Colloq. Math., 104 (2006), no. 2, 223-283. MR.2197078(2006m:37009) 
14. E. Glasner and M. Megrelishvili, New algebras of functions on topological groups arising from G-spaces, Fundamenta Math., 201 (2008), 1-51. MR2439022(2010f:37015)

15. E. Glasner and M. Megrelishvili, On fixed point theorems and nonsensitivity, Israel J. of Math. (to appear).

16. E. Glasner, M. Megrelishvili and V.V. Uspenskij, On metrizable enveloping semigroups, Israel J. of Math. 164 (2008), 317-332. MR2391152 (2009a:37013)

17. E. Glasner and B. Weiss, Sensitive dependence on initial conditions, Nonlinearity 6, (1993), 1067-1075. MR 1251259 (94j:58109)

18. G. Godefroy, Compacts de Rosenthal, Pacific J. Math., 91 (1980), 293-306. MR615679 (82f:54030)

19. M.M. Guillermo, Indice de K-determinación de espacios topológicos y $\sigma$-fragmentabilidad de aplicaciones, Tesis Doctoral, Universidad de Murcia, Spain, 2003.

20. W. Huang, Tame systems and scrambled pairs under an abelian group action, Ergod. Th. Dynam. Sys. 26 (2006), 1549-1567. MR.2266373 (2007j:37012)

21. R.C. James, A separable somewhat reflexive Banach space with nonseparable dual, Bull. Amer. Math. Soc., 80 (1974), 738-743. MR0417763 (54:5811)

22. J.E. Jayne, J. Orihuela, A.J. Pallares and G. Vera, $\sigma$-fragmentability of multivalued maps and selection theorems, J. Funct. Anal. 117 (1993), no. 2, 243-273. MR1244937 (94m:46023)

23. J.E. Jayne and C.A. Rogers, Borel selectors for upper semicontinuous set-valued maps, Acta Math. 155 (1985), 41-79. MR793237 (87a:28011)

24. A.S. Kechris, Classical descriptive set theory, Graduate Texts in Mathematics, 156, SpringerVerlag, 1991. MR 1321597 (96e:03057)

25. D. Kerr and H. Li, Independence in topological and $C^{*}$-dynamics, Math. Ann. 338 (2007), 869-926. MR2317754 (2009a:46126)

26. A. Köhler, Enveloping semigrops for flows, Proceedings of the Royal Irish Academy, 95A (1995), 179-191. MR.1660377(99i:47056)

27. J. Lindenstrauss and C. Stegall, Examples of separable spaces which do not contain $l_{1}$ and whose duals are nonseparable, Studia Math., 54 (1975), 81-105. MR0390720 (52:11543)

28. M. Megrelishvili, Fragmentability and continuity of semigroup actions, Semigroup Forum, $\mathbf{5 7}$ (1998), 101-126. MR:1621881 (99c:54053)

29. M. Megrelishvili, Operator topologies and reflexive representability, In: "Nuclear groups and Lie groups" Research and Exposition in Math. series, vol. 24, Heldermann Verlag, Berlin, 2001, 197-208. MR 1858149 (2002m:46027)

30. M. Megrelishvili, Fragmentability and representations of flows, Topology Proceedings, 27:2 (2003), 497-544. See also: www.math.biu.ac.il/ ${ }^{\sim}$ megereli. MR2077804 (2005h:37042)

31. E. Michael and I. Namioka, Barely continuous functions, Bull. Acad. Polon. Sci. Ser. Sci. Math. Astron. Phys., 24 (1976), 889-892. MR0431092 (55:4094)

32. I. Namioka, Separate continuity and joint continuity, Pacific. J. Math., 51 (1974), 515-531. MR 0370466 (51:6693)

33. I. Namioka, Radon-Nikodým compact spaces and fragmentability, Mathematika 34, (1987), 258-281. MR933504 (89i:46021)

34. I. Namioka and R.R. Phelps, Banach spaces which are Asplund spaces, Duke Math. J., 42 (1975), 735-750. MR0390721 (52:11544)

35. E. Odell and H. P. Rosenthal, A double-dual characterization of separable Banach spaces containing $l^{1}$, Israel J. Math., 20 (1975), 375-384. MR0377482 (51:13654)

36. R. Pol, Note on Compact Sets of First Baire Class Functions, Proc. Amer. Math. Soc. 96, No. 1. (1986), pp. 152-154. MR813828 (87b:54033)

37. L.H. Riddle, E. Saab and J.J. Uhl, Sets with the weak Radon-Nikodým property in dual Banach spaces, Indiana Univ. Math. J., 32 (1983), 527-541. MR703283 (84h:46028)

38. H.P. Rosenthal, A characterization of Banach spaces containing $l_{1}$, Proc. Nat. Acad. Sci. U.S.A., 71 (1974), 2411-2413. MR0358307 (50:10773)

39. H.P. Rosenthal, Point-wise compact subsets of the first Baire class, Amer. J. of Math., 99:2 (1977), 362-378. MR0438113 (55:11032)

40. H.P. Rosenthal, Some recent discoveries in the isomorphic theory of Banach spaces, Bull. Amer. Math. Soc. 84:5 (1978), 803-831. MR499730 (80d:46023)

41. E. Saab and P. Saab, A dual geometric characterization of Banach spaces not containing $l_{1}$, Pacific J. Math., 105:2 (1983), 413-425. MR691612 (85c:46013) 
42. M. Talagrand, Pettis integral and measure theory, Mem. Amer. Math. Soc. No. 51 (1984). MR756174 (86j:46042)

43. S. Todorčević, Topics in topology, Lecture Notes in Mathematics, 1652, Springer-Verlag, 1997. MR.1442262 (98g:54002)

Department of Mathematics, Tel-Aviv University, Tel Aviv, Israel

E-mail address: glasner@math.tau.ac.il

URL: http://www.math.tau.ac.il/ glasner

Department of Mathematics, Bar-Ilan University, 52900 Ramat-Gan, Israel

E-mail address: megereli@math.biu.ac.il

URL: http://www.math.biu.ac.il/ megereli 